Ann. Scient. Éc. Norm. Sup.,

$4^{\text {e }}$ série, t. 39, 2006, p. 301 à 333.

\title{
RELATIVE KAZHDAN PROPERTY
}

\author{
BY YVES DE CORNULIER
}

\begin{abstract}
We perform a systematic investigation of Kazhdan's relative Property (T) for pairs $(G, X)$, where $G$ is a locally compact group and $X$ is any subset. When $G$ is a connected Lie group or a $p$-adic algebraic group, we provide an explicit characterization of subsets $X \subset G$ such that $(G, X)$ has relative Property (T). In order to extend this characterization to lattices $\Gamma \subset G$, a notion of "resolutions" is introduced, and various characterizations of it are given. Special attention is paid to subgroups of $\operatorname{SU}(2,1)$ and $\mathrm{SO}(4,1)$.

(C) 2006 Elsevier SAS
\end{abstract}

RÉSUMÉ. - Nous faisons une étude systématique de la notion de propriété (T) relative (de Kazhdan) pour des paires $(G, X)$, où $G$ est un groupe localement compact et $X$ une partie quelconque. Lorsque $G$ est un groupe de Lie connexe ou un groupe algébrique $p$-adique, nous caractérisons de façon explicite les parties $X \subset G$ telles que $(G, X)$ a la propriété $(\mathrm{T})$ relative. Une notion convenable de «résolutions » permet d'étendre ces résultats aux réseaux $\Gamma \subset G$, et nous en donnons diverses caractérisations. Une attention particulière est portée aux sous-groupes de $\mathrm{SU}(2,1)$ et $\mathrm{SO}(4,1)$.

(c) 2006 Elsevier SAS

\section{Introduction}

Kazhdan's Property (T) was introduced in a short paper by Kazhdan [24] in 1967. Since then, many consequences and characterizations have been given by various authors.

The notion of relative Property for a pair $(G, N)$, where $N$ is a normal subgroup in $G$ was implicit in Kazhdan's paper, and later made explicit by Margulis [28]. The case when $H$ is an abelian normal subgroup is, by far, the best understood [24,28,9,35,36]. However, it seems that it was initially only considered as a technical tool. The most famous case is the following: in order to prove Property $(\mathrm{T})$ for $\mathrm{SL}_{3}(\mathbf{R})$ (and other higher rank algebraic groups over local fields), one uses, in most proofs, Property (T) for the pair $\left(\mathrm{SL}_{2}(\mathbf{R}) \ltimes \mathbf{R}^{2}, \mathbf{R}^{2}\right)$.

The definition of relative Property (T) has been extended in [22] to pairs $(G, H)$ with $H$ not necessarily normal in $G$. Such pairs are extensively used by Popa (see [34] and the references therein), in the context of operator algebras. Popa's work motivated, for instance, new examples of group pairs with relative Property (T) of the form $(G \ltimes N, N)$, with $N$ abelian [39,19].

We extend the definition of relative Property ( $\mathrm{T})$ to pairs $(G, X)$, where $X$ is any subset of $G$. The motivation for this is that, given $G$, the knowledge of the family of subsets $X$ such that $(G, X)$ has relative Property (T) contains much information about the unitary dual of $G$. It provides a knowledge of $G$ much more precise than the bare information whether $G$ has Property (T). On the other hand, the family of subgroups with relative Property (T) provides a strictly weaker information (see Example 1.12). 
Let $G$ be a locally compact group, and let $X \subset G$ be any subset. We say that $(G, X)$ has relative Property (T) if for every net $\left(\varphi_{i}\right)$ of positive definite functions on $G$ that converges to 1 uniformly on compact subsets, the convergence is uniform on $X$.

In Section 2 we establish various characterizations of relative Property (T) for a pair $(G, X)$, which were already known [23] in the case when $X$ is a subgroup. Here are the main ones (the relevant definitions are recalled at the beginning of Section 2).

THEOREM 1.1 (See Theorems 2.2.3, 2.3.2, and 2.3.3). - Let $G$ be a locally compact, $\sigma$-compact group, and let $X \subset G$ be any subset. The following are equivalent:

(1) (Positive definite functions) $(G, X)$ has relative Property (T).

(2) (Representations with almost invariant vectors) For every $\varepsilon>0$, and for every unitary representation $\pi$ of $G$ that almost has invariant vectors, $\pi$ has $(X, \varepsilon)$-invariant vectors.

(3) (Kazhdan pairs) For every $\varepsilon>0$, there exist a compact subset $K \subset G$ and $\eta>0$ such that every unitary representation of $G$ that has a $(K, \eta)$-invariant vector has a $(X, \varepsilon)$-invariant vector.

(4) (Conditionally negative definite functions) Every conditionally negative definite function on $G$ is bounded on $X$.

(5) (Isometric actions on affine Hilbert spaces) For every affine, isometric action of $G$ on an affine Hilbert space $\mathscr{H}$, and every $v \in \mathscr{H}, X v$ is bounded.

(6) (Topology of the unitary dual) For every $\varepsilon>0$, and for every net $\left(\pi_{i}\right)$ of irreducible unitary representations of $G$ that converges to $1_{G}$, eventually $\pi_{i}$ has a $(X, \varepsilon)$-invariant vector.

Recall that a locally compact group $G$ is Haagerup if it has a net of $C_{0}$ positive definite functions that converges to 1 , uniformly on compact subsets. It is clear from the definition that if $G$ is Haagerup, then, for every $X \subset G$, the pair $(G, X)$ has relative Property (T) if and only if $X$ is relatively compact in $G$. The question whether the converse holds was asked (in a slightly different formulation) in [2]. We say that $G$ satisfies the TH alternative if it is either Haagerup, or has a subset $X$ with noncompact closure, such that $(G, X)$ has relative Property (T). The question becomes: does there exist a locally compact group that does not satisfy the TH alternative? We leave it open.

By a result of Kazhdan, if $G$ is a locally compact group with Property (T), then $G$ is compactly generated. The same argument shows that if $(G, X)$ has Property (T), then $X$ is contained in a compactly generated subgroup of $G$. Here is a stronger result, which says, in a certain sense, that all questions about relative Property (T) reduce to the compactly generated case.

THEOREM 1.2 (Theorem 2.5.2). - Let $G$ be a locally compact group, and let $X \subset G$ be any subset. Then $(G, X)$ has relative Property $(\mathrm{T})$ if and only if there exists an open, compactly generated subgroup $H$ of $G$, containing $X$, such that $(H, X)$ has relative Property $(\mathrm{T})$.

Note that Theorem 1.2 is new even in the case when $X \subset G$ is a normal subgroup. As a corollary of Theorem 1.2 (see Remark 2.1.7), we deduce that a locally compact group satisfies the TH alternative if and only if all its open, compactly generated subgroup do so.

We are interested in the question of determining, given a group $G$, subsets $X$ such that $(G, X)$ has relative Property (T). As a general result, we show, provided that $G$ is compactly generated, that such subsets coincide with the bounded subsets for a well-defined, essentially left-invariant metric on $G$, which we call the $H$-metric (see Section 2.6).

In Section 3, we focus on relative Property $(\mathrm{T})$ in connected Lie groups and linear algebraic groups over a local field $\mathbf{K}$ of characteristic zero.

Let $G$ be a connected Lie group. Let $R$ be its radical, and $S$ a Levi factor. Define $S_{n c}$ as the sum of all non-compact factors of $S$, and $S_{n h}$ as the sum of all factors of $S_{n c}$ with Property (T).

$4^{\text {e }}$ SÉRIE - TOME $39-2006-\mathrm{N}^{\circ} 2$ 
Finally define the T-radical $R_{T}=\overline{S_{n h}\left[S_{n c}, R\right]}$. It is easily checked to be a characteristic subgroup of $G$.

THEOREM 1.3 (Theorem 3.3.1). - $\left(G, R_{T}\right)$ has relative Property (T).

On the other hand, by results in [10, Chapter 4], $G / R_{T}$ has the Haagerup Property. As a consequence:

Corollary 1.4. - Let $X \subset G$ be any subset, and p denote the projection $G \rightarrow G / R_{T}$. Then $(G, X)$ has relative Property $(\mathrm{T})$ if and only if $\overline{p(X)}$ is compact.

The proof of Theorem 1.3 makes use of the following proposition:

PROPOSITION 1.5. - Let $S$ be a semisimple connected Lie group without compact factors, and $R$ a nilpotent, simply connected Lie group, endowed with an action of $S$, and set $G=S \ltimes R$. Suppose that $[S, R]=R$. Then $(G, R)$ has relative Property $(\mathrm{T})$.

The proposition is proved as follows: we work by induction on $\operatorname{dim}(R)$, we pick a nontrivial central subgroup $V$ of $R$, normal in $G$, and we can reduce to two cases. Either $[S, V]=V$, so that, by well-known results (which can be attributed to Kazhdan, Margulis, Burger), the pair $(G, V)$ has relative Property (T), and the result follows by induction, or $V$ is central in $G$. To handle this case, we formulate an ad-hoc result of stability of relative Property (T) by central extensions.

The case of a linear algebraic group $G$ over a local field $\mathbf{K}$ of characteristic zero is similar. Let $R$ be the radical, $S$ a Levi factor, and define $S_{n c}$ and $S_{n h}$ as in the case of Lie groups, and set $R_{T}=S_{n h}\left[S_{n c}, R\right]$; this is a closed characteristic subgroup.

THEOREM 1.6 (Theorem 3.2.4). - $\left(G(\mathbf{K}), R_{T}(\mathbf{K})\right)$ has relative Property (T).

On the other hand, it is easily checked that $G / R_{T}$ has the Haagerup Property. A corollary similar to Corollary 1.4 follows.

Another corollary is the following result, already known:

COROLLARY 1.7. - Let $G$ be a connected Lie group (respectively a linear algebraic group over $\mathbf{K})$.

(1) $G$ [respectively $G(\mathbf{K})]$ has Property (T) if and only if $R_{T}$ [respectively $\left.R_{T}(\mathbf{K})\right]$ is cocompact in $G$ [respectively in $G(\mathbf{K})]$.

(2) $G$ [respectively $G(\mathbf{K})]$ is Haagerup if and only if $R_{T}=1$.

Assertion (1) is a result of S.P. Wang [40], and (2) is due to [10, Chapter 4] for connected Lie groups and to [12] in the $p$-adic case.

Section 4 is devoted to explain how these phenomena are inherited by subgroups of finite covolume.

Let $G$ be a locally compact group, $N$ a closed, normal subgroup, and $H$ a subgroup of finite covolume in $G$. It is known (see [23]) that if $(G, N)$ has relative Property (T), then so does $(H, N \cap H)$. However, this result is of limited use insofar as $N \cap H$ may be small (for instance, reduced to $\{1\}$ ) even if $N$ is noncompact: this phenomenon is very frequent in the context of irreducible lattices in products of algebraic groups over local fields.

We need a definition that enlarges the notion of relative Property (T) of normal subgroups. The datum of $N$ normal in $G$ is equivalent to the datum of the morphism $G \rightarrow G / N$. More generally, we are led to consider arbitrary locally compact groups $G, Q$, and a morphism $f: G \rightarrow Q$ with dense image. We say that $f$ is a resolution if, for every unitary representation $\pi$ of $G$ almost having invariant vectors, $\pi$ has a nonzero subrepresentation $\rho$ factoring through a representation $\tilde{\rho}$ of $Q$, and $\tilde{\rho}$ almost has invariant vectors. 
Given a closed normal subgroup $N$ in a locally compact group $G,(G, N)$ has relative Property (T) if and only if $G \rightarrow G / N$ is a resolution. In view of Theorems 1.3 and 1.6, a wealth of examples of resolutions are provided by the following result, essentially due to Margulis [29, Chapter III, Section 6], and which also uses arguments borrowed from [4].

THEOREM 1.8 (Theorem 4.3.1). - Let $G$ be a locally compact group, $N$ a closed, normal subgroup. Suppose that $(G, N)$ has relative Property (T) (equivalently, the projection $p: G \rightarrow$ $G / N$ is a resolution).

Let $H$ be a closed subgroup of finite covolume in $G$, and write $Q=\overline{p(H)}$. Then $p: H \rightarrow Q$ is a resolution.

Resolutions allow to prove compact generation of some locally compact groups. The following theorem generalizes Proposition 2.8 of [26].

THEOREM 1.9 (Theorem 4.2.8). - Let $G \rightarrow Q$ be a resolution. Then $G$ is compactly generated if and only if $Q$ is so.

Thus, compact generation can be said to be "invariant under resolutions". We provide some other examples.

Proposition 1.10 (See Theorem 4.2.8, Corollary 4.7.10, Proposition 4.5.2). - Let $G \rightarrow Q$ be a resolution. Then, if $(\mathrm{P})$ is one of the properties below, then $G$ has Property $(\mathrm{P})$ if and only if $Q$ does so.

- Property (T);

- Compact generation;

- Property (FA): every isometric action on a tree has a fixed point;

- Every isometric action on a Euclidean space has a fixed point;

- Property $(\tau)$ : the trivial representation is isolated among irreducible unitary representations with finite image.

Resolutions give rise to pairs with relative Property (T).

Proposition 1.11. - Let $p: H \rightarrow Q$ be a resolution. Given any subset $X \subset H$, if $(Q, p(X))$ has relative Property $(\mathrm{T})$, then so does $(H, X)$. In particular, if $Q$ is Haagerup, then $(H, X)$ has relative Property $(\mathrm{T})$ if and only if $\overline{p(X)}$ is compact.

The first consequence is that if $Q$ is Haagerup, then $H$ satisfies the TH alternative (Corollary 4.2.7). In view of Theorems 1.6 and 1.8, this applies to lattices in products of real and $p$-adic algebraic groups.

We can also derive some new phenomena related to relative Property (T).

Example 1.12 (See Proposition 4.5.5, the proof of Proposition 4.6.3(2), and Remark 4.6.4). Consider the group $\Gamma_{n}=\mathrm{SO}_{n}\left(\mathbf{Z}\left[2^{1 / 3}\right]\right) \ltimes \mathbf{Z}\left[2^{1 / 3}\right]^{n}$.

If $n \leqslant 2$, it is solvable, hence Haagerup. If $n=3,4$, it was observed in [12] that $\Gamma_{n}$ is not Haagerup, but has no infinite subgroup with relative Property (T). Thanks to resolutions, we can see this more concretely: the natural morphism $i: \Gamma_{n} \rightarrow \mathrm{SO}_{n}\left(\mathbf{Z}\left[2^{1 / 3}\right]\right) \ltimes \mathbf{R}^{n}$ is a resolution. It follows that $\left(\Gamma_{n}, B_{n}\right)$ has relative Property $(\mathrm{T})$, where $B_{n}$ is the intersection of the unit ball of $\mathbf{R}^{n}$ with $\mathbf{Z}\left[2^{1 / 3}\right]^{n}$.

If $n \geqslant 5, \Gamma_{n}$ has a different behaviour, due to the fact that $\mathrm{SO}_{n}\left(\mathbf{Z}\left[2^{1 / 3}\right]\right)$ has Property (T) (whereas it is Haagerup if $n \leqslant 4$ ). It follows that, if $n \geqslant 5$, then $\Gamma_{n} \rightarrow \mathrm{SO}_{n}(\mathbf{R}) \ltimes \mathbf{R}^{n}$ is a resolution. We deduce an interesting property for $\Gamma_{n}$ : it has Property $(\tau)$, but has a finitedimensional unitary representation $\pi$ such that $H^{1}\left(\Gamma_{n}, \pi\right) \neq 0$.

$4^{\mathrm{e}}$ SÉRIE - TOME $39-2006-\mathrm{N}^{\circ} 2$ 
As pointed out in [33], all previously known examples of group pairs with relative Property (T) were derived from group pairs where the subgroup is normal. For instance, for every group $G$, $\left(\left(\mathrm{SL}_{2}(\mathbf{Z}) \ltimes \mathbf{Z}^{2}\right) * G, \mathbf{Z}^{2}\right)$ has relative Property $(\mathrm{T})$; the only role of $G$ is to prevent $\mathbf{Z}^{2}$ from being normal.

Resolutions and Proposition 1.11 allows us to find group pairs with relative Property (T) that do not derive in such a way from group pairs with a normal subgroup. This is illustrated by projections of irreducible lattices from $\mathrm{SO}(4,1) \times \mathrm{SO}(5, \mathbf{C})$, such as $\mathrm{SO}(4,1)\left(\mathbf{Z}\left[2^{1 / 3}\right]\right)$, see Theorem 4.6.1 and the proof of Proposition 4.6.3(1).

Proposition 1.13. - Let $G$ be either $\mathrm{SO}(4,1)$ or $\mathrm{SU}(2,1)$, and $\Gamma$ a subgroup, viewed as a discrete group (but not necessarily discrete in $G$ ).

(1) If $\Lambda \subset \Gamma$ is a normal subgroup and $(\Gamma, \Lambda)$ has relative Property $(\mathrm{T})$, then $\Lambda$ is finite.

(2) There exists $\Gamma$ containing an infinite subgroup $\Lambda$ such that $(\Gamma, \Lambda)$ has relative Property $(\mathrm{T})$.

Finally, we prove various equivalences for resolutions. They generalize known equivalences for pairs $(G, N)$ with $N$ normal in $G$, but the proofs are more involved.

THEOREM 1.14 (See Theorems 4.7.6 and 4.7.11). - Let $G, Q$ be a locally compact $\sigma$-compact groups, and let $f: G \rightarrow Q$ be a morphism with dense image. The following are equivalent:

(1) $f: G \rightarrow Q$ is a resolution.

(2) $f$ is an "affine resolution": for every isometric affine action of $G$ on a Hilbert space, there exists a $G$-invariant nonempty closed affine subspace on which the action factors through $Q$.

(3) For every subset $X \subset G$ such that $\overline{f(X)}$ is compact, $(G, X)$ has relative Property (T).

(4) For every net $\left(\pi_{i}\right)$ of irreducible unitary representations of $G$, if $\pi_{i} \rightarrow 1_{G}$, then eventually $\pi_{i}$ factors through a representation $\tilde{\pi}_{i}$ of $Q$, and $\tilde{\pi}_{i} \rightarrow 1_{Q}$.

\section{Property (T) relative to subsets}

TERMINOLOGY. - Throughout the paper, by morphism between topological groups we mean continuous group homomorphisms. If $X$ is a Hausdorff topological space, a subset $Y \subset X$ is relatively compact if its closure in $X$ is compact.

If $G$ is a group, a positive definite function on $G$ is a function: $\varphi: G \rightarrow \mathbf{C}$ such that $\varphi(g)=\overline{\varphi\left(g^{-1}\right)}$ for all $g \in G$, and, for all $n \in \mathbf{N},\left(g_{1}, \ldots, g_{n}\right) \in G^{n}$ and $\left(c_{1}, \ldots, c_{n}\right) \in \mathbf{C}^{n}$, the inequality $\sum_{i, j} c_{i} \overline{c_{j}} \varphi\left(g_{i}^{-1} g_{j}\right) \geqslant 0$ holds. We say that a positive definite function $\varphi$ is normalized if $\varphi(1)=1$.

If $G$ is a group, a conditionally negative definite function on $G$ is a function: $\psi: G \rightarrow \mathbf{R}$ such that $\psi(1)=0, \psi(g)=\psi\left(g^{-1}\right)$ for all $g \in G$, and for all $n \in \mathbf{N},\left(g_{1}, \ldots, g_{n}\right) \in G^{n}$ and $\left(c_{1}, \ldots, c_{n}\right) \in \mathbf{R}^{n}$ such that $\sum c_{i}=0$, the inequality $\sum_{i, j} c_{i} c_{j} \psi\left(g_{i}^{-1} g_{j}\right) \leqslant 0$ holds.

A locally compact group $G$ is Haagerup if for every compact $K \subset G$ and $\varepsilon>0$, there exists a real-valued normalized positive definite function in $G$, vanishing at infinity, and such that $\left.\varphi\right|_{K}>1-\varepsilon$. A locally compact group $G$ is $a-T$-menable if it has a proper conditionally negative definite function. These two properties are introduced and called (3A) and (3B) in [2], where the authors prove their equivalence in the $\sigma$-compact case.

All unitary representations $\pi$ of a locally compact group are supposed continuous, that is, the function $g \mapsto \pi(g) \xi$ is continuous for every $\xi$ in the representation space. Recall that $1_{G} \prec \pi$ (or $1 \prec G$ if there is no ambiguity) means that the unitary representation $\pi$ almost has invariant vectors, that is, for every compact subset $K \subset G$ and every $\varepsilon>0$, there exists a nonzero vector $\xi$ such that $\sup _{g \in K}\|\pi(g) \xi-\xi\| \leqslant \varepsilon\|\xi\|$. 


\subsection{Property (T) relative to subsets}

DEFINITION 2.1.1. - Let $G$ be a locally compact group, and $X$ any subset. We say that $(G, X)$ has relative Property (T) if, for every net $\left(\varphi_{i}\right)$ of continuous normalized positive definite functions that converges to 1 uniformly on compact subsets, the convergence is uniform on $X$.

We say that $(G, X)$ has relative Property (FH) if every continuous conditionally definite negative function on $G$ is bounded on $X$.

If $\varphi$ is a positive definite function on $G$, then so is $|\varphi|^{2}$. Thus, the definition of relative Property (T) remains unchanged if we only consider real-valued positive definite functions or even non-negative real-valued positive definite functions.

Question 2.1.2 (See [2]). - For a locally compact group $G$, consider the two following properties.

(1) $G$ is a-T-menable;

(2) for every subset $X \subset G$, the pair $(G, X)$ has relative Property (FH) if and only if $\bar{X}$ is compact.

(Note that the implication (1) $\Rightarrow(2)$ is trivial.) Does there exist a $\sigma$-compact, locally compact group $G$ satisfying (2) and not (1)?

Remark 2.1.3. - If $G$ is locally compact but not $\sigma$-compact, (1) and (2) of Question 2.1.2 are not equivalent: (1) is always false, while a characterization of (2) is less clear. For instance, if $G$ is any abelian locally compact group, then (2) is fulfilled. On the other hand, it was shown in [13] that if $F$ is a non-nilpotent finite group, then $F^{\mathbf{N}}$, viewed as a discrete group, does not satisfy (2); moreover, if $F$ is perfect, then $F^{\mathbf{N}}$ has Property (FH). Besides, being locally finite, these groups are amenable, hence Haagerup.

It is maybe worth comparing Question 2.1.2 to the following result, essentially due to [21]:

PROPOSITION 2.1.4. - The locally compact, $\sigma$-compact group $G$ is a-T-menable if and only if there exists a sequence $\left(\psi_{n}\right)_{n \in \mathbf{N}}$ of continuous conditionally negative definite functions on $G$, such that, for every sequence $\left(M_{n}\right)$ of positive numbers, $\left\{g \in G \mid \forall n, \psi_{n}(g) \leqslant M_{n}\right\}$ is compact.

Proof. - The direct implication is trivial (take any proper function $\psi$, and $\psi_{n}=\psi$ for all $n$ ). Conversely, suppose the existence of a family $\left(\psi_{n}\right)$ satisfying the condition. Let $\left(K_{n}\right)$ be an increasing sequence of compact subsets of $G$ whose interiors cover $G$. There exists a sequence $\left(\varepsilon_{n}\right)$ such that $\varepsilon_{n} \psi_{n} \leqslant 2^{-n}$ on $K_{n}$. Set $\psi=\sum_{n} \varepsilon_{n} \psi_{n}$; since the series is convergent uniformly on compact subsets, $\psi$ is well-defined and continuous. Then, for every $M<\infty$, the set $\{\psi \leqslant M\}$ is contained in $\left\{g \mid \forall n, \psi_{n} \leqslant M / \varepsilon_{n}\right\}$, which is, by assumption, compact.

Question 2.1.5. - Does there exist a locally compact group $G$ without the Haagerup Property, but with no unbounded subset $X$ such that $(G, X)$ has relative Property $(\mathrm{T})$ ?

Remark 2.1.6. - Akemann and Walter [1] introduce the following definition: a locally compact group has the weak dual Riemann-Lebesgue Property if, for every $\varepsilon, \eta>0$ and every compact subset $K$ of $G$, there exists a compact subset $\Omega$ of $G$ such that, for every $x \in G-\Omega$, there exists a normalized, real-valued, positive definite function $\varphi$ on $G$ such that $\varphi(x) \leqslant \eta$ and $\sup \{1-\varphi(g) \mid g \in K\} \leqslant \varepsilon$.

It can be shown [14, Proposition 2.7] that a locally compact group $G$ has the weak-dual Riemann-Lebesgue if and only if every subset $X \subset G$ such that $(G, X)$ has relative Property (T) is relatively compact. Akemann and Walker [2] ask if the weak dual Riemann-Lebesgue Property is equivalent to the Haagerup Property; this question is therefore equivalent to Question 2.1.5.

$4^{\mathrm{e}}$ SÉRIE - TOME $39-2006-\mathrm{N}^{\circ} 2$ 
Remark 2.1.7. - (1) It follows from Theorem 2.2.3 that Questions 2.1.2 and 2.1.5 are equivalent for locally compact, $\sigma$-compact groups.

(2) It follows from [10, Proposition 6.1.1] and Theorem 2.5.2 that if Question 2.1.5 has a positive answer, then the example can be chosen compactly generated.

DEFINITION 2.1.8. - We say that $G$ satisfies the TH alternative if it is either Haagerup, or has a subset $X$ with noncompact closure, such that $(G, X)$ has relative Property (T).

Question 2.1.5 becomes: does there exist a locally compact group not satisfying the TH alternative?

Remark 2.1.9.-Here is an obstruction to the Haagerup Property for a locally compact, compactly generated group $G$, that does not formally imply the existence of a non-relatively compact subset with relative Property (T). Let $\omega$ belong to the Stone-Čech boundary $\beta G \backslash G$ of $G$. Let us say that $(G, \omega)$ has relative Property (T) if, for every conditionally negative definite function $\psi$ on $G$, its canonical extension $\tilde{\psi}: \beta G \rightarrow \mathbf{R}_{+} \cup\{\infty\}$ satisfies $\tilde{\psi}(\omega)<\infty$.

It is clear that relative Property (T) for $(G, \omega)$ prevents $G$ from being Haagerup. On the other hand, I see no reason why this should imply the existence of a non-relatively compact subset with relative Property $(\mathrm{T})$.

\subsection{Various equivalences}

DEFINITION 2.2.1. - Let $G$ be a locally compact group and $X \subset G$. Given a unitary representation $\pi$ of $G$ and $\varepsilon \geqslant 0$, a $(X, \varepsilon)$-invariant vector for $\pi$ is a nonzero vector $\xi$ in the representation space such that $\|\pi(g) \xi-\xi\| \leqslant \varepsilon\|\xi\|$ for every $g \in X$.

Definition 2.2.2. - Let $G$ be a locally compact group, $X, W$ subsets, and $\varepsilon, \eta>0$. We say that $(W, \eta)$ is a $\varepsilon$-Kazhdan pair for $(G, X)$ if, for every unitary representation $\pi$ of $G$ that has a $(W, \eta)$-invariant vector, $\pi$ has a $(X, \varepsilon)$-invariant vector. Given $G, X, W, \varepsilon$, if such $\eta>0$ exists, we say that $W$ is a $\varepsilon$-Kazhdan subset for $(G, X)$.

The following result generalizes a result due to Jolissaint [23] when $X$ is a subgroup.

THEOREM 2.2.3. - Let $G$ be a locally compact group, and let $X \subset G$ be a subset. Consider the following properties:

(1) $(G, X)$ has relative Property $(\mathrm{T})$.

(2) For every $\varepsilon>0$, there exists a compact $\varepsilon$-Kazhdan subset for $(G, X)$.

$\left(2^{\prime}\right)$ For some $\varepsilon<\sqrt{2}$, there exists a compact $\varepsilon$-Kazhdan subset for $(G, X)$.

(3) For every $\varepsilon>0$ and every unitary representation $\pi$ of $G$ such that $1 \prec \pi$, the representation $\pi$ has a $(X, \varepsilon)$-invariant vector.

$\left(3^{\prime}\right)$ There exists $\varepsilon<\sqrt{2}$ such that for every unitary representation $\pi$ of $G$ satisfying $1 \prec \pi$, the representation $\pi$ has a $(X, \varepsilon)$-invariant vector.

(4) $(G, X)$ has relative Property $(\mathrm{FH})$, i.e. satisfies (5) of Theorem 1.1.

Then the following implications hold:

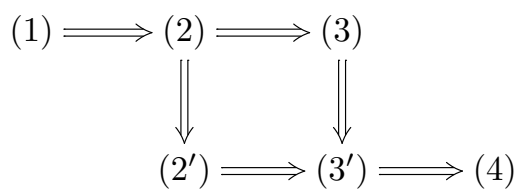

Moreover, if $G$ is $\sigma$-compact, then (4) $\Rightarrow(1)$, so that they are all equivalent. 
Proof. $-(1) \Rightarrow(2)$ Suppose the contrary. There exists $\varepsilon>0$ such that, for every $\eta>0$ and every compact subset $K \subset G$, there exists a unitary representation $\pi_{\eta, K}$ of $G$ that has a $(K, \eta)$ invariant unit vector $\xi_{\eta, K}$, but has no $(X, \varepsilon)$-invariant vector. Denote by $\varphi_{\eta, K}$ the corresponding coefficient. Then, when $\eta \rightarrow 0$ and $K$ becomes big, $\varphi_{\eta, K}$ converges to 1 , uniformly on compact subsets. By relative Property (T), the convergence is uniform on $X$. It follows that, for some $K$ and some $\eta$, the representation $\pi_{\eta, K}$ has a $\varepsilon$-invariant vector, a contradiction.

$(2) \Rightarrow\left(2^{\prime}\right),(2) \Rightarrow(3),\left(2^{\prime}\right) \Rightarrow\left(3^{\prime}\right)$, and $(3) \Rightarrow\left(3^{\prime}\right)$ are immediate.

$\left(3^{\prime}\right) \Rightarrow(4)$ Let $\psi$ be a conditionally negative definite function on $G$, and, for $t>0$, let $\left(\pi_{t}, \mathcal{H}_{t}\right)$ be the cyclic unitary representation of $G$ associated with the positive definite function $e^{-t \psi}$. Set $\rho_{t}=\pi_{t} \otimes \pi_{t}$. Since $\pi_{t} \rightarrow 1_{G}$ when $t \rightarrow 0$, so does $\rho_{t}$.

Suppose that $\psi$ is not bounded on $X: \psi\left(x_{n}\right) \rightarrow \infty$ for some sequence $\left(x_{n}\right)$ in $X$. Then we claim that for every $t>0$ and every $\xi \in \mathcal{H}_{t} \otimes \overline{\mathcal{H}_{t}}$, we have $\left\langle\rho_{t}\left(x_{n}\right) \xi, \xi\right\rangle \rightarrow 0$ when $n \rightarrow \infty$. Equivalently, for every $\xi \in \mathcal{H}_{t} \otimes \overline{\mathcal{H}_{t}}$ of norm one, $\left\|\rho_{t}\left(x_{n}\right) \xi-\xi\right\| \rightarrow \sqrt{2}$. This is actually established in the proof of [23, Lemma 2.1] (where the assumption that $X=H$ is a subgroup is not used for this statement).

By Lebesgue's dominated convergence theorem, it follows that if $\rho$ denotes the representation $\bigoplus_{t>0} \rho_{t}$, then $\left\langle\rho\left(x_{n}\right) \xi, \xi\right\rangle \rightarrow 0$ for every $\xi$. In particular, for every $\varepsilon<\sqrt{2}$, the representation $\rho$ has no $(X, \varepsilon)$-invariant vector. Since $1 \prec \rho$, this contradicts $\left(3^{\prime}\right)$.

$(4) \Rightarrow(1)$ The proof is a direct adaptation of that of the analogous implication in [2, Theorem 3] and we do not repeat it here.

Remark 2.2.4. - When $X=H$ is a subgroup, we retrieve a result of [23]. Note that, in this case, by a well-known application of the "Lemma of the centre" [3, Lemma 2.2.7], Condition (2') of Theorem 2.2.3 can be chosen with $\varepsilon=0$, i.e. becomes: for every unitary representation of $G$ such that $1 \prec \pi$, there exists a nonzero $H$-invariant vector.

Remark 2.2.5. - When $G$ is not $\sigma$-compact, whether the implication $\left(3^{\prime}\right) \Rightarrow(1)$ holds is not known, except when $X$ is a normal subgroup [23]. On the other hand, it is known [13] that (4) $\Rightarrow$ $\left(3^{\prime}\right)$ does not always hold for general locally compact groups, even when we assume that $X=G$ is a discrete group.

\subsection{Relative Property ( $T$ ) can be read on irreducible unitary representations}

The following lemma, due to Choquet (unpublished), is proved in [17, B.14, p. 355].

LEMMA 2.3.1. - Let $K$ be a compact, convex subset of a locally convex space E. Let $x$ be an extremal point of $K$. Let $\mathcal{W}$ be the set of all open half-spaces of $E$ that contain $x$. Then $\{W \cap K \mid W \in \mathcal{W}\}$ is a neighbourhood basis of $x$ in $K$.

Denote $\mathcal{P}(G)$ [respectively $\mathcal{P}_{1}(G)$, respectively $\mathcal{P}_{\leqslant 1}(G)$ ] the set of all (complex-valued) positive definite function $\varphi$ on $G$ [respectively such that $\varphi(1)=1$, respectively such that $\varphi(1) \leqslant 1]$.

Recall that $\varphi \in \mathcal{P}(G)$ is pure if it satisfies one of the two equivalent conditions: (i) $\varphi$ is associated to an irreducible unitary representation; (ii) $\varphi$ belongs to an extremal axis of the convex cone $\mathcal{P}(G)$.

THEOREM 2.3.2. - Let $G$ be a locally compact group and $X$ a subset. The following are equivalent:

(i) $(G, X)$ has relative Property (T).

(ii) For every net of continuous, normalized pure positive definite functions on $G$ converging to 1 , the convergence is uniform on $X$.

$4^{\text {e }}$ SÉRIE - TOME $39-2006-\mathrm{N}^{\circ} 2$ 
Proof. - (i) $\Rightarrow$ (ii) is trivial; conversely suppose that $G$ satisfies (ii). Endow the space $L^{\infty}(G)=L^{1}(G)^{*}$ with the weak* topology. Let $\mathcal{W}$ be the set of all open half-spaces of $L^{\infty}(G)$ containing the constant function 1 . Finally set $K=\mathcal{P}_{\leqslant 1}(G)$.

Recall Raikov's Theorem [17, Théorème 13.5.2]: on $\mathcal{P}_{1}(G)$, the weak* topology coincides with the topology of uniform convergence on compact subsets. Let $\mathcal{L}$ be the set of all continuous linear forms $u$ on $L^{\infty}(G)$ such that $u(1)=1$ and $\left.u\right|_{K} \leqslant 1$. Since $K$ is convex and compact for the weak*-topology, by Lemma 2.3.1, $\{\{u>1-\varepsilon\} \cap K \mid u \in \mathcal{L}, \varepsilon>0\}$ is a basis of open neighbourhoods of 1 in $\mathcal{P}_{\leqslant 1}(G)$.

Hence, by (ii), and using Raikov's Theorem, for every $1>\varepsilon>0$, there exist $u \in \mathcal{L}$ and $\eta>0$ such that, for every pure $\varphi \in \mathcal{P}_{1}(G), u(\varphi)>1-\eta$ implies $\varphi \geqslant 1-\varepsilon$ on $X$.

Let $\varphi=\sum \lambda_{i} \varphi_{i}$ be a convex combination of continuous, normalized, pure positive definite functions $\varphi_{i}$. Suppose that $u(\varphi)>1-\eta \varepsilon$. Decompose $\varphi$ as $\sum \lambda_{j} \varphi_{j}+\sum \lambda_{k} \varphi_{k}$, where $u\left(\varphi_{j}\right)>$ $1-\eta$ and $u\left(\varphi_{k}\right) \leqslant 1-\eta$. Then

$$
\begin{aligned}
1-\eta \varepsilon & <u(\varphi)=\sum \lambda_{j} u\left(\varphi_{j}\right)+\sum \lambda_{k} u\left(\varphi_{k}\right) \\
& \leqslant \sum \lambda_{j}+\sum \lambda_{k}(1-\eta)=1-\eta \sum \lambda_{k},
\end{aligned}
$$

so that $\sum \lambda_{k} \leqslant \varepsilon$. Hence, on $X$, we have

$$
\begin{aligned}
\varphi & =\sum \lambda_{j} \varphi_{j}+\sum \lambda_{k} \varphi_{k} \geqslant \sum \lambda_{j}(1-\varepsilon)-\sum \lambda_{k} \\
& \geqslant \sum \lambda_{j}-2 \varepsilon \geqslant 1-3 \varepsilon .
\end{aligned}
$$

Set $K_{u, \varepsilon \eta}=\{\varphi \in K \mid u(\varphi)>1-\varepsilon \eta\}$, and $K_{c p}=\{\varphi \in K \mid \varphi$ is a convex combination of continuous, normalized pure positive definite functions on $G\}$. By [3, Theorem C.5.5], $K_{c p}$ is weak* dense in $\mathcal{P}_{1}(G)$. Since $K_{u, \varepsilon \eta}$ is open in $K$, this implies that $K_{c p} \cap K_{u, \varepsilon \eta}$ is weak*-dense in $\mathcal{P}_{1}(G) \cap K_{u, \varepsilon \eta}$. By Raikov's Theorem, it is also dense for the topology of uniform convergence on compact subsets. Hence, since for all $\varphi \in K_{c p} \cap K_{u, \varepsilon \eta}, \varphi \geqslant 1-3 \varepsilon$ on $X$, the same holds for all $\varphi \in \mathcal{P}_{1}(G) \cap K_{u, \varepsilon \eta}$.

THEOREM 2.3.3. - Let $G$ be a locally compact, $\sigma$-compact group. The following are equivalent:

(1) $(G, X)$ has relative Property $(\mathrm{T})$.

(2) For every $\varepsilon>0$, there exists a neighbourhood $V$ of $1_{G}$ in $\widehat{G}$ such that every $\pi \in V$ has a $(X, \varepsilon)$-invariant vector.

$\left(2^{\prime}\right)$ For some $\varepsilon<\sqrt{2}$, there exists a neighbourhood $V$ of $1_{G}$ in $\widehat{G}$ such that every $\pi \in V$ has $a(X, \varepsilon)$-invariant vector.

Proof. $-(2) \Rightarrow\left(2^{\prime}\right)$ is trivial.

$\left(2^{\prime}\right) \Rightarrow(1)$. By a result of Kakutani and Kodaira [11, Theorem 3.7], there exists a compact normal subgroup $K$ of $G$ such that $G / K$ is second countable. So we can suppose that $G$ is second countable. Consider a unitary representation $\pi$ of $G$ almost having invariant vectors. Arguing as in [16, proof of Lemme 1], $\pi$ contains a nonzero subrepresentation entirely supported by $V$. We conclude by Lemma 2.3.5 below that $\pi$ has a $\varepsilon^{\prime}$-invariant vector, where $\varepsilon<\varepsilon^{\prime}<\sqrt{2}$. This proves that $\left(3^{\prime}\right)$ of Theorem 2.2.3 is satisfied.

$(1) \Rightarrow(2)$. This is immediate from Condition (2) in Theorem 2.2.3.

Remark 2.3.4. - The special case when $X$ is a subgroup is claimed without proof in [22, Chapter 1, 18].

Let $G$ be a second countable, locally compact group, and $X \subset G$. Let $(Z, \mu)$ be measured space, with $\mu(Z)>0$ and $\mu \sigma$-finite. Let $\left(\left(\mathcal{H}_{z}\right)_{z \in Z}, \Gamma\right)$ be a measurable field of Hilbert 
spaces [17, A 69], $\Gamma$ denoting a space of measurable vector fields. Let $\left(\pi_{z}\right)$ be a field of unitary representations, meaning that $z \mapsto \pi_{z}(g) x(z)$ is measurable, for every $x \in \Gamma, g \in G$. Recall that, by definition, there exists a sequence $\left(x_{n}\right)$ in $\Gamma$ such that, for every $z \in Z$, the family $\left(x_{n}(z)\right)$ is total in $\mathcal{H}_{z}$. Set $\pi=\int^{\oplus} \pi_{z} d \mu(z)$.

Lemma 2.3.5. - Fix $\varepsilon>0$. Suppose that, for every $z, \pi_{z}$ has $a(X, \varepsilon)$-invariant vector. Then $\pi$ has a $\left(X, \varepsilon^{\prime}\right)$-invariant vector for every $\varepsilon^{\prime}>\varepsilon$.

Proof. - Fix $0<\eta<1$. First note that replacing the family $\left(x_{n}\right)$ by the family of all its rational combinations if necessary, we can suppose that, for every $z \in Z$ and every $v \in \mathcal{H}_{z}$ of norm one, there exists $n$ such that $\left\|v-x_{n}(z)\right\| \leqslant \eta$. In particular, if $v$ is $(X, \varepsilon)$-invariant, then, for all $x \in X$, $\left\|\pi_{z}(x) x_{n}(z)-x_{n}(z)\right\| \leqslant \varepsilon+2 \eta$ and $\left\|x_{n}(z)\right\| \geqslant\|1-\eta\|$, so that $x_{n}(z)$ is $(X,(\varepsilon+2 \eta) /(1-\eta))$ invariant. Now define, for all $n \geqslant 0$,

$$
A_{n}=\left\{z \in Z \mid 1-\eta \leqslant\left\|x_{n}(z)\right\| \leqslant 1+\eta, x_{n}(z) \text { is }(X,(\varepsilon+2 \eta) /(1-\eta)) \text {-invariant }\right\} .
$$

We have $\bigcup A_{n}=Z$ by the remark above. Using that $X$ is separable, it is immediate that $A_{n}$ is measurable for every $n$. Accordingly, there exists $n_{0}$ such that $\mu\left(A_{n_{0}}\right)>0$. Using that $\mu$ is $\sigma$-finite, there exists a measurable subset $B \subset A_{n_{0}}$ such that $0<\mu(B)<\infty$. Define $\xi$ as the field

$$
z \mapsto \begin{cases}x_{n_{0}}(z), & z \in B \\ 0, & \text { otherwise. }\end{cases}
$$

Then it is clearly measurable, and

$$
\|\xi\|^{2}=\int_{B}\left\|x_{n_{0}}(z)\right\|^{2} d \mu(z) \geqslant(1-\eta)^{2} \mu(B),
$$

and, for every $g \in X$,

$$
\begin{aligned}
\|\pi(g) \xi-\xi\|^{2} & =\int_{B}\left\|\pi_{z}(g) x_{n_{0}}(z)-x_{n_{0}}(z)\right\|^{2} d \mu(z) \\
& \leqslant\left((\varepsilon+2 \eta)^{2}(1+\eta)^{2} /(1-\eta)^{2}\right) \mu(B) .
\end{aligned}
$$

It follows that $\xi \neq 0$ and is $\left(X,(\varepsilon+2 \eta)(1+\eta) /(1-\eta)^{2}\right)$-invariant. Finally, for every $\varepsilon^{\prime}>\varepsilon$, we can choose $\eta$ sufficiently small so that $(\varepsilon+2 \eta)(1+\eta) /(1-\eta)^{2} \leqslant \varepsilon^{\prime}$.

\subsection{Some stability results}

We note for reference the following immediate but useful result:

Proposition 2.4.1. - Let $G$ be a locally compact group and $X_{1}, \ldots, X_{n}$ be subsets. Denote by $X_{1} \cdots X_{n}$ the pointwise product $\left\{x_{1} \cdots x_{n} \mid\left(x_{1}, \ldots, x_{n}\right) \in X_{1} \times \cdots \times X_{n}\right\}$. Suppose that, for every $i,\left(G, X_{i}\right)$ has relative Property $(\mathrm{T})$ [respectively $\left.(\mathrm{FH})\right]$. Then $\left(G, X_{1} \cdots X_{n}\right)$ has relative Property (T) [respectively $(\mathrm{FH})]$.

Proof. - It suffices to prove the case when $n=2$, since then the result follows by induction. For the case of Property (FH), this follows from the inequality $\psi(g h)^{1 / 2} \leqslant \psi(g)^{1 / 2}+\psi(h)^{1 / 2}$ for every conditionally negative definite function $\psi$. For the case of Property (T), a similar inequality holds since, if $\varphi$ is normalized positive definite, then $1-|\varphi|^{2}$ is conditionally negative definite.

$4^{\text {e }}$ SÉRIE - TOME $39-2006-\mathrm{N}^{\circ} 2$ 
Example 2.4.2. - (1) If $G_{1}, \ldots, G_{n}$ are locally compact groups, and $X_{i} \subset G_{i}$, and if $\left(G_{i}, X_{i}\right)$ has relative Property (T) [respectively $(\mathrm{FH})]$ for every $i$, then $\left(\prod G_{i}, \prod X_{i}\right)$ also has relative Property (T) [respectively (FH)].

(2) Let $G$ be a group, $H_{1}$ a subgroup, and $H_{2}$ a subgroup of finite index in $H_{1}$. If $\left(G, H_{2}\right)$ has relative Property (T), then so does $\left(G, H_{1}\right)$. It suffices to apply Proposition 2.4.1 to $n=2$, $X_{1}=H_{2}$, and $X_{2}$ a finite transversal of $H_{1}$ modulo $H_{2}$.

(3) Fix $n \geqslant 3$, let $A$ a topologically finitely generated locally compact commutative ring, and set $G=\mathrm{SL}_{n}(A)$. Denote by $V_{n, m}$ the elements in $G$ that are products of $\leqslant m$ elementary matrices. Then it follows from [35, Corollary 3.5] that $\left(G, V_{n, m}\right)$ has relative Property (T) for all $m$. It is not known whether, for such $A$, there exists $m$ such that $V_{n, m}=E_{n}(A)$, the subgroup generated by elementary matrices; this seems to be an open question whenever $A$ is a finitely generated commutative ring of Krull dimension $\geqslant 2$, for instance $A=\mathbf{Z}[X]$, or $A=\mathbf{F}_{p}[X, Y]$.

The following proposition is trivial.

Proposition 2.4.3. - Let $G$ be a locally compact group, $H$ a closed subgroup, and $Y \subset$ $X \subset H$ subsets. If $(H, X)$ has relative Property $(\mathrm{T})[$ respectively $(\mathrm{FH})]$, then so does $(G, Y)$.

Proposition 2.4.4 (Stability by extensions). - Let $G$ be a locally compact group, $N$ a closed normal subgroup, and $X \subset G$ a subset. Denote by $p: G \rightarrow G / N$ the projection.

If $(G, N)$ and $(G / N, p(X))$ have relative Property (T) [respectively $(\mathrm{FH})]$, then so does $(G, X)$.

Proof. - The assertion about relative Property (FH) is immediate; that about relative Property $(\mathrm{T})$ is straightforward, using the following fact [3, Lemma B.1.1]: for every compact subset $K$ of $G / N$, there exists a compact subset $\widetilde{K}$ of $G$ such that $p(\widetilde{K})=K$.

\subsection{Relative Property (T) and compact generation}

It is well known that a locally compact group with Property (T) is compactly generated. We generalize this result. The following lemma is the easy part of such a generalization; we are going to use it to prove something stronger.

LEMMA 2.5.1. - Let $G$ be a locally compact group, and let $X \subset G$ be a subset such that $(G, X)$ has relative Property $(\mathrm{T})$. Then $X$ is contained in an open, compactly generated subgroup of $G$.

Proof. - For every open, compactly generated subgroup $\Omega$ of $G$, let $\lambda_{\Omega}$ be the quasi-regular representation of $G$ on $\ell^{2}(G / \Omega)$. Let $\delta_{\Omega} \in \ell^{2}(G / \Omega)$ be the Dirac function on $G / \Omega$. Let $\varphi_{\Omega}$ be the corresponding coefficient. Then $\varphi_{\Omega}$ tends to 1 , uniformly on compact subsets, when $\Omega$ becomes big. By relative Property (T), the convergence is uniform on $X$, so that, for some $\Omega$, we have $\left|1-\varphi_{\Omega}\right|<1$ on $X$. Since $\varphi_{\Omega}$ has values in $\{0,1\}$, this implies that $\delta_{\Omega}=\delta_{g \Omega}$ for all $g \in X$, that is, $g \in \Omega$. Hence $X \subset \Omega$.

The following theorem shows that, in a certain sense, all the information about relative Property (T) lies within compactly generated subgroups.

THEOREM 2.5.2. - Let $G$ be a locally compact group, and $X \subset G$ a subset. Then $(G, X)$ has relative Property $(\mathrm{T})$ if and only if there exists an open, compactly generated subgroup $H$ such that $X \subset H$ and $(H, X)$ has relative Property $(\mathrm{T})$.

LEMMA 2.5.3. - Fix $0<\varepsilon<1$. Let $G$ be a locally compact group and $X$ a subset. Then $(G, X)$ has relative Property $(\mathrm{T})$ if and only if, for every net $\left(\varphi_{i}\right)$ of normalized, real-valued 
continuous positive definite functions converging to 1 uniformly on compact subsets, eventually $\left|\varphi_{i}\right|>\varepsilon$ on $X$.

Proof. - The forward implication is trivial. Suppose that $(G, X)$ does not have relative Property (T). Then there exists a net $\left(\varphi_{i}\right)$ of normalized, real-valued continuous positive definite functions converging to 1 uniformly on compact subsets, such that $\alpha=\sup _{i} \inf _{g \in X} \varphi_{i}(g)<1$. Then, for some $n \in \mathbf{N}, \alpha^{n}<\varepsilon$. Hence, $\left(\varphi_{i}^{n}\right)$ is a net of normalized, continuous positive definite functions that converges to 1 uniformly on compact subsets, but, for no $i,\left|\varphi_{i}^{n}\right|>\varepsilon$ on $X$.

Proof of Theorem 2.5.2. - By Lemma 2.5.1, there exists $\Omega \supset X$ an open, compactly generated subgroup. Let $\left(K_{i}\right)$ be an increasing net of open, relatively compact subsets, covering $G$, and denote by $H_{i}$ the subgroup generated by $K_{i}$. We can suppose that $\Omega \subset H_{i}$ for all $i$.

Suppose by contradiction that, for every $i,\left(H_{i}, X\right)$ does not have Property $(\mathrm{T})$. Then, using Lemma 2.5.3, for all $i$ and all $n$, there exists a normalized, continuous positive definite function $\varphi_{i, n}$ on $H_{i}$, such that $\varphi_{i, n} \geqslant 1-2^{-n}$ on $K_{i}$ and $\inf _{X} \varphi_{i, n} \leqslant 1 / 2$. Since $H_{i}$ is open in $G$, we can extend $\varphi_{i, n}$ to all of $G$, by sending the complement of $H_{i}$ to 0 . It is clear that the net $\left(\varphi_{i, n}\right)$ tends to 1 uniformly on compact subsets of $G$, but $\inf _{X} \varphi_{i, n} \leqslant 1 / 2$. This contradicts that $(G, X)$ has relative Property $(\mathrm{T})$.

\section{6. $H$-metric}

First recall that a length function on a group $G$ is a function $L: G \rightarrow \mathbf{R}_{+}$satisfying the subadditivity condition $L(g h) \leqslant L(g)+L(h)$ for all $g, h$, and such that $L(1)=0$ and $L(g)=$ $L\left(g^{-1}\right)$ for all $g \in G$. A length function defines a (maybe non-separated) left-invariant metric on $G$ by setting $d(g, h)=L\left(g^{-1} h\right)$.

Observe that if $L_{1}, L_{2}$ are two length functions, then so is $L=\max \left(L_{1}, L_{2}\right)$. Indeed, we can suppose $L_{1}(g h) \geqslant L_{2}(g h)$. Then $L(g h)=L_{1}(g h) \leqslant L_{1}(g)+L_{1}(h) \leqslant L(g)+L(h)$.

Also observe that a pointwise limit of length functions is a length function. If follows that the upper bound of a family of length functions, provided that it is everywhere finite, is a length function.

Now let $G$ be a locally compact, compactly generated group, and $K$ a relatively compact, open generating subset. Define $\Psi_{K}$ as the upper bound of all (continuous, real-valued) conditionally negative definite functions $\psi$ such that $\psi \leqslant 1$ on $K$. Recall that if $\psi$ is a real-valued conditionally negative definite function, then $\psi^{1 / 2}$ is a length function. It follows that $\Psi_{K}^{1 / 2}$ is a length function. It is easily checked that it defines a separated metric on $G$, whose closed balls are closed (for the initial topology). We call it the $H$-metric. It is easy to observe that if $K$ and $L$ are two open, relatively compact generating subsets, then there exist constants $\lambda, \lambda^{\prime}>0$ such that $\lambda \Psi_{K} \leqslant \Psi_{L} \leqslant \lambda^{\prime} \Psi_{K}$. Accordingly, the identity map defines a bi-Lipschitz map between these two metrics, and the choice of $K$ is not essential at all.

Proposition 2.6.1. - Let $G$ be a locally compact, compactly generated group, and $X$ a subset. Then $(G, X)$ has relative Property $(\mathrm{T})$ if and only if $X$ is bounded for the $H$-metric.

Proof. - First recall that, since $G$ is $\sigma$-compact, relative Property (T) and relative Property (FH) are equivalent by Theorem 2.2.3.

If $X$ is bounded for the $H$-metric, and $\psi$ is a (continuous, real-valued) conditionally negative definite function on $X$, then, for some constant $\alpha>0, \alpha \psi \leqslant 1$ on $K$. So $\psi \leqslant \alpha^{-1} \Psi_{K}$, which is bounded on $X$, and thus $(G, X)$ has relative Property (FH).

Conversely, suppose that $X$ is not bounded for the $H$-metric. Then there exist a sequence of (continuous, real-valued) conditionally negative definite functions $\psi_{n}$, bounded by 1 on $K$, and a sequence $x_{n}$ of $X$ such that $\psi_{n}\left(x_{n}\right) \geqslant 4^{n}$. Set $\psi=\sum 2^{-n} \psi_{n}$. Since the convergence

$4^{e}$ SÉRIE - TOME $39-2006-\mathrm{N}^{\circ} 2$ 
is uniform on compact subsets, $\psi$ is a well-defined continuous conditionally negative definite function on $G$, and $\psi\left(x_{n}\right) \geqslant 2^{n}$, so that $\psi$ is not bounded on $X$, and $(G, X)$ does not have relative Property $(\mathrm{FH})$.

COROLLARY 2.6.2. - Let $G$ be a locally compact, compactly generated group.

(1) G has Property (T) is and only if it is bounded for the H-metric.

(2) G has no non-compact closed subset with relative Property (T) if and only if $G$ is proper for the H-metric (that is, the balls for the H-metric are compact for the initial topology).

It is maybe interesting comparing the $H$-metric with the word metric (relative to any compact generating set). A general result in this direction has been obtained by V. Lafforgue [25]: if $G$ does not have Property (T), if $L_{K}$ denotes the word length with respect to the compact generating set $K$, then there exists a conditionally negative definite function $\psi$ on $G$ satisfying $\left.\psi\right|_{K} \leqslant 1$ and

$$
\sup \left\{\psi(x)^{1 / 2} \mid x \in G \text { and } L_{K}(x) \leqslant n\right\} \geqslant \frac{\sqrt{n}}{2}-2 .
$$

In particular, if $H_{K}=\Psi_{K}^{1 / 2}$ is the length in the $H$-metric, then, for every $0<C<1 / 2$, there exists a sequence $\left(x_{n}\right)$ in $G$ such that, for all $n, L_{K}\left(x_{n}\right) \leqslant n$ and $H_{K}\left(x_{n}\right) \geqslant C \sqrt{n}$.

On the other hand, R. Tessera [38] has proved that, for a polycyclic group $\Gamma$, the $H$-metric is equivalent to the word metric, although it is known that $\Gamma$ has no conditionally negative definite function with quadratic growth unless $\Gamma$ is virtually abelian [15].

\section{Relative Property (T) in connected Lie groups and $p$-adic algebraic groups}

\subsection{Preliminaries}

Given a locally compact group $G$, we can naturally raise the problem of determining for which subsets $X$ the pair $(G, X)$ has relative Property (T). Here is a favourable case, where the problem is completely solved.

LEMMA 3.1.1. - Let $G$ be a locally compact group, and $N$ a normal subgroup such that $(G, N)$ has relative Property $(\mathrm{T})$ and $G / N$ is Haagerup. Let $X$ be any subset of $G$. Then $(G, X)$ has relative Property (T) if and only if the image of $X$ in $G / N$ is relatively compact.

Proof. - The condition is clearly necessary, since relative Property (T) is inherited by images.

Conversely, if the image of $X$ in $G / N$ is relatively compact, there exists a compact subset $K$ of $G$ such that $X$ is contained in $K N=\{k n \mid(k, n) \in K \times N\}$. Let $\psi$ be a continuous, conditionally negative definite function on $G$. Then $\psi$ is bounded on $N$ and on $K$, hence on $K N$, hence on $X$. This proves that $(G, X)$ has relative Property (FH). In view of Theorem 2.2.3, this is sufficient if $G$ is $\sigma$-compact. Actually, we can reduce to this case: indeed, by Theorem 2.5.2, there exists an open, compactly generated subgroup $H$ of $G$, that contains $N$ and can be supposed to contain $K$, such that $(H, N)$ has relative Property (T).

Recall the key result, due to Shalom [36, Theorem 5.5] (see also [3, §1.4]).

Proposition 3.1.2. - Let $G$ be a locally compact group and $N$ a closed normal abelian subgroup. Assume that the only mean on the Borel subsets of the Pontryagin dual $\widehat{N}=$ $\operatorname{Hom}(N, \mathbf{R} / \mathbf{Z})$, invariant under the action of $G$ by conjugation, is the Dirac measure at zero. Then the pair $(G, N)$ has relative Property $(\mathrm{T})$. 
This result allows to prove relative Property (T) for certain normal abelian subgroups. Since we also deal with nilpotent subgroups, we use the following proposition, which generalizes [10, Proposition 4.1.4].

PROPOSITION 3.1.3. - Let $G$ be a locally compact, $\sigma$-compact group, $N$ a closed subgroup, and let $Z$ be a closed, central subgroup of $G$ contained in $[N, N]$. Suppose that every morphism of $N$ into a compact Lie group has an abelian image.

Suppose that the pair $(G / Z, N / Z)$ has Property (T). Then $(G, N)$ has Property (T).

Proof. - It suffices to show that $(G, Z)$ has relative Property (T). Indeed, since the pairs $(G, Z)$ and $(G / Z, N / Z)$ have relative Property (T), it then follows by Proposition 2.4.4 that $(G, N)$ has relative Property $(T)$.

We use an argument similar to the proof of [40, Lemma 1.6]. To show that $(G, Z)$ has relative Property (T), we use the characterization by nets of irreducible unitary representations (see Theorem 2.3.3). Let $\pi_{i}$ be a net of irreducible unitary representations of $G$ converging to the trivial representation: we must show that eventually $\pi_{i}$ factors through $Z$. Let $\bar{\pi}_{i}$ be the contragradient representation of $\pi_{i}$. Then $\pi_{i} \otimes \overline{\pi_{i}}$ converges to the trivial representation. By irreducibility, $\pi_{i}$ is scalar in restriction to $Z$, hence $\pi_{i} \otimes \bar{\pi}_{i}$ is trivial on $Z$, so factors through $G / Z$. Since $(G / Z, N / Z)$ has Property (T), the restriction to $N$ of $\pi_{i} \otimes \overline{\pi_{i}}$ eventually contains the trivial representation. By a standard argument [3, Appendix 1], this means that $\left.\pi_{i}\right|_{N}$ eventually contains a finite-dimensional subrepresentation $\rho_{i}$.

Remark that $\overline{\rho_{i}(N)}$ is a compact Lie group; so it is, by assumption, abelian. This means that $[N, N]$ acts trivially; hence $Z$ does so as well: $\rho_{i}$ is trivial on $Z$. Hence, for large $i, \pi_{i}$ has nonzero $Z$-invariants vectors; by irreducibility, $\pi_{i}$ is trivial on $Z$. Accordingly $(G, Z)$ has Property (T).

We shall use the following well-known result of Furstenberg [20].

THEOREM 3.1.4 (Furstenberg). - Let $\mathbf{K}$ be a local field, $V$ a finite-dimensional $K$-vector space. Let $G \subset \mathrm{PGL}(V)$ be a Zariski connected (but not necessarily Zariski closed) subgroup, whose closure is not compact. Suppose that $G$ preserves a probability measure $\mu$ on the projective space $\mathbf{P}(V)$. Then there exists a $G$-invariant proper projective subspace $W \subsetneq \mathbf{P}(V)$ such that $\mu(W)=1$.

Remark 3.1.5. - Observe that a subgroup of $\mathrm{PGL}(V)$ preserves an invariant mean on $\mathbf{P}(V)$ if and only if it preserves a probability: indeed, a mean gives rise to a normalized positive linear form on $L^{\infty}(\mathbf{P}(V))$, and restricts to a normalized positive linear form on $C(\mathbf{P}(V))$, defining a probability.

We say that a topological group $G$ is discompact ${ }^{1}$ if there is no nontrivial morphism of $G$ to a compact group.

Remark 3.1.6. - If $G$ is a discompact locally compact group, then it has trivial abelianization. Indeed, it follows that its abelianization is also discompact, so has trivial Pontryagin dual, so it trivial by Pontryagin duality.

The following corollary is an easy consequence of Theorem 3.1.4 (see [14, Corollary 2.39] for a proof).

COROLlaRY 3.1.7. - Let $G$ be a discompact locally compact group. Let $V$ be a finitedimensional vector space over $\mathbf{K}$, and let $G \rightarrow \mathrm{GL}(V)$ be any continuous representation. Then $G$ preserves a probability on $\mathbf{P}(V)$ if and only if $G$ has a nonzero fixed point on $V$.

\footnotetext{
${ }^{1}$ This is often called "minimally almost periodic", but we prefer the terminology "discompact", introduced in [36].

$4^{\mathrm{e}}$ SÉRIE - TOME $39-2006-\mathrm{N}^{\circ} 2$
} 
Example 3.1.8. - (1) Let $G$ be a simply connected, simple group over $\mathbf{K}$, of positive $\mathbf{K}$-rank. Then $G(\mathbf{K})$ is discompact. Indeed, $G(\mathbf{K})$ is generated by elements whose conjugacy classes contain 1 in their closure: this follows from the following observations: $G(\mathbf{K})$ is simple [29, Chapter I, Theorem 1.5.6 and Theorem 2.3.1(a)], and there exists a subgroup of $G$ isomorphic to either $\mathrm{SL}_{2}(\mathbf{K})$ or $\mathrm{PSL}_{2}(\mathbf{K})$ [29, Chapter I, Proposition 1.6.3]. Accordingly, every morphism of $G(\mathbf{K})$ into a compact group has trivial image.

(2) Let $G$ be a connected, noncompact, simple Lie group. Then $G$ is discompact. Indeed, such a group is generated by connected subgroups locally isomorphic to $\mathrm{SL}_{2}(\mathbf{R})$, hence is generated by elements whose conjugacy class contains 1 .

Proposition 3.1.9. - Let $V$ be a finite-dimensional space over a local field $\mathbf{K}$. Let $G$ be any locally compact group, and $\rho: G \rightarrow \mathrm{GL}(V)$ a continuous representation. Then $(G \ltimes V, V)$ has relative Property (T) if and only if $G$ preserves no probability on $\mathbf{P}\left(V^{*}\right)$. In particular, if $G$ is discompact, then $(G \ltimes V, V)$ has relative Property (T) if and only if $G$ fixes no point in $V^{*}$.

Proof. - Suppose, by contradiction that $G$ preserves a probability on $\mathbf{P}\left(V^{*}\right)$ and $(G \ltimes V, V)$ has relative Property (T). By Theorem 3.1.4, the finite index subgroup $G_{0}$ of $G$ (its unit component in the inverse image of the Zariski topology from $\mathrm{GL}(V)$ ) preserves a nonzero subspace $W \subset V^{*}$, such that the image of the morphism $G_{0} \rightarrow \operatorname{PGL}(W)$ has compact closure. Since $W$ is a subspace of $V^{*}, W^{*}$ is a quotient of $V$. By Corollary 4.1(2) in [23], $\left(G_{0} \ltimes V, V\right)$ has relative Property (T), and so has $\left(G_{0} \ltimes W^{*}, W^{*}\right)$. This implies that $\left(\overline{\rho\left(G_{0}\right)} \ltimes W^{*}, W^{*}\right)$ also has relative Property (T). But $\overline{\rho\left(G_{0}\right)} \ltimes W^{*}$ is amenable, so that $W^{*}$ is compact, and this is a contradiction.

The converse is due to M. Burger [9, Proposition 7]. It is obtained by combining Proposition 3.1.2 and Remark 3.1.5. The second assertion follows from Corollary 3.1.7.

Remark 3.1.10. - It is worth noting that, in Proposition 3.1.9, and in view of Corollary 3.1.7, relative Property (T) for $(G \ltimes V, V)$ only depends on the closure (for the ordinary topology) of the image of $G$ in $\operatorname{PGL}(V)$.

\subsection{Relative Property (T) in algebraic groups over local fields of characteristic zero}

We denote by $\mathbf{K}$ a local field of characteristic zero. Here is the main lemma of this paragraph.

LEMMA 3.2.1. - Let $G$ be a linear algebraic $\mathbf{K}$-group, which decomposes as $S \ltimes R$, where $S$ is semisimple and $\mathbf{K}$-isotropic, and $R$ is unipotent.

Suppose that $[S, R]=R$. Then $(G(\mathbf{K}), R(\mathbf{K}))$ has relative Property $(\mathrm{T})$.

Proof. - Replacing $S$ by its universal cover if necessary, we can suppose that $S$ is simply connected. We then argue by induction on the dimension of $R$. If the dimension is zero, there is nothing to prove; suppose $R \neq 1$. Let $Z$ be the last nonzero term of its descending central series.

First case: $Z$ is central in $G$. The hypothesis $[S, R]=R$ implies that $R$ is not abelian. Hence $Z \subset[R, R]$, so that $Z(\mathbf{K}) \subset[R, R](\mathbf{K})$. By [6, Lemma 13.2] $[R, R](\mathbf{K})=[R(\mathbf{K}), R(\mathbf{K})]$, so that $Z(\mathbf{K}) \subset[R(\mathbf{K}), R(\mathbf{K})]$. We must check that the hypotheses of Proposition 3.1.3 are fulfilled. Let $W$ be a compact Lie group, and $R(\mathbf{K}) \rightarrow W$ a morphism with dense image: we must show that $W$ is abelian. Since $R(\mathbf{K})$ is solvable, the connected component $W_{0}$ is abelian. Moreover, $R(\mathbf{K})$ is divisible, so $W / W_{0}$ is also divisible; this implies $W=W_{0}$. Accordingly, by Proposition 3.1.3, since $(G(\mathbf{K}) / Z(\mathbf{K}), R(\mathbf{K}) / Z(\mathbf{K}))$ has relative Property (T) by induction hypothesis, it follows that $(G(\mathbf{K}), R(\mathbf{K}))$ has relative Property (T).

Second case: $Z$ is not central in $G$. Set $N=[S, Z]$. Then $[S, N]=N$. By Proposition 3.1.9 and in view of Example 3.1.8(1), $(G(\mathbf{K}), N(\mathbf{K}))$ has relative Property (T). By the induction 
assumption, $\left((G / N)(\mathbf{K}),\left(R_{u} / N\right)(\mathbf{K})\right)$, which coincides with $\left(G(\mathbf{K}) / N(\mathbf{K}), R_{u}(\mathbf{K}) / N(\mathbf{K})\right)$, has relative Property (T). Hence $\left(G(\mathbf{K}), R_{u}(\mathbf{K})\right)$ has relative Property (T).

Let $G$ be a linear algebraic group over $\mathbf{K}$. We denote by $R_{u}$ its unipotent radical, and $L$ a reductive Levi factor (so that $G_{0}=L \ltimes R_{u}$ ). We decompose $L$ as an almost product $L_{m} L_{n m}$, where $L_{m}$ (respectively $L_{n m}$ ) includes the centre of $L$, and the simple factors of rank zero (respectively includes the simple factors of positive rank). ${ }^{2}$

Let $R$ be the radical of $G$, let $S$ be a Levi factor, and decompose it as $S_{c} S_{n c}$, where $S_{c}$ (respectively $S_{n c}$ ) is the sum of all factors of rank 0 (respectively of positive rank).

If $\mathfrak{g}$ is a Lie algebra and $\mathfrak{h}_{1}, \mathfrak{h}_{2}$ are two subspaces, we denote by $\left[\mathfrak{h}_{1}, \mathfrak{h}_{2}\right]$ (respectively $\left[\mathfrak{h}_{1}, \mathfrak{h}_{2}\right]_{v}$ ) the Lie algebra (respectively the subspace) generated by the elements of the form $\left[h_{1}, h_{2}\right]$, $\left(h_{1}, h_{2}\right) \in \mathfrak{h}_{1} \times \mathfrak{h}_{2}$.

LEMMA 3.2.2. - For every Levi factors $L, S$ of respectively $R_{u}$ and $R$, we have $\left[L_{n m}, R_{u}\right]=$ $\left[S_{n c}, R\right]$, and this is a $\mathbf{K}$-characteristic subgroup of $G$.

Proof. - We can work within the Lie algebra. We first justify that $\left[\mathfrak{l}_{n m}, \mathfrak{r}_{u}\right]$ is an ideal: indeed,

$$
\left[\mathfrak{l},\left[\mathfrak{l}_{n m}, \mathfrak{r}_{u}\right]\right] \subset\left[\left[\mathfrak{l}, \mathfrak{l}_{n m}\right], \mathfrak{r}_{u}\right]+\left[\mathfrak{l}_{n m},\left[\mathfrak{l}, \mathfrak{r}_{u}\right]\right] \subset\left[\mathfrak{l}_{n m}, \mathfrak{r}_{u}\right]
$$

since $\left[\mathfrak{l}, \mathfrak{l}_{n m}\right] \subset \mathfrak{l}_{n m}$ and $\left[\mathfrak{l}, \mathfrak{r}_{u}\right] \subset \mathfrak{r}_{u}$. On the other hand,

$$
\begin{aligned}
{\left[\mathfrak{r}_{u},\left[\mathfrak{l}_{n m}, \mathfrak{r}_{u}\right]\right] } & =\left[\mathfrak{r}_{u},\left[\mathfrak{l}_{n m},\left[\mathfrak{l}_{n m}, \mathfrak{r}_{u}\right]\right]\right] \\
& \subset\left[\mathfrak{l}_{n m},\left[\mathfrak{r}_{u},\left[\mathfrak{l}_{n m}, \mathfrak{r}_{u}\right]\right]\right]+\left[\left[\mathfrak{l}_{n m}, \mathfrak{r}_{u}\right],\left[\mathfrak{l}_{n m}, \mathfrak{r}_{u}\right]\right] \\
& \subset\left[\mathfrak{l}_{n m}, \mathfrak{r}_{u}\right] .
\end{aligned}
$$

It follows that $\left[L_{n m}, R_{u}\right]$ is a normal subgroup of $G$. By [5, (5.1)], the $\mathbf{K}$-conjugacy class of $L$ does not depend of the choice of $L$. So the same thing holds for $L_{n m}$ (which is $\mathbf{K}$-characteristic in $L$ ). Accordingly, $\left[L_{n m}, R_{u}\right]$ is a $\mathbf{K}$-characteristic subgroup of $G$.

Now, since $S_{n c}$ is a reductive $\mathbf{K}$-subgroup of $G$, again using [5, (5.1)], up to $\mathbf{K}$-conjugate if necessary, we can suppose that $S_{n c} \subset L$, so that finally $S_{n c}=L_{n m}$, and $R=L_{r} \ltimes R_{u}$, where $L_{r}$ is the unit component of centre of $L$. Since $\left[L_{n c}, L_{r}\right]=1$, we obtain $\left[S_{n c}, R\right]=\left[L_{n c}, R\right]=$ $\left[L_{n c}, R_{u}\right]$.

Let $S_{n h}$ be the sum of all simple factors $H$ of $S_{n c}$ such that $H(\mathbf{K})$ is not Haagerup (equivalently: has Property $(\mathrm{T})$ ): these are factors of rank $\geqslant 2$, and also, when $\mathbf{K}=\mathbf{R}$, factors locally isomorphic to $\operatorname{Sp}(n, 1)$ or $F_{4(-20)}$.

Definition 3.2.3. - Define $R_{T}$ as the K-subgroup $S_{n h}\left[S_{n c}, R\right]$ of $G$.

THEOREM 3.2.4. $-R_{T}$ is a $\mathbf{K}$-characteristic subgroup of $G$, the quotient group $G(\mathbf{K}) / R_{T}(\mathbf{K})$ is Haagerup, and $\left(G(\mathbf{K}), R_{T}(\mathbf{K})\right)$ has relative Property $(\mathrm{T})$.

Proof. - It follows from Lemma 3.2.2 that $\left[S_{n c}, R\right]$ is unipotent. Consider the $\mathbf{K}$-subgroup $W=S_{n c}\left[S_{n c}, R\right]$ of $G$. Applying Lemma 3.2.1 to $W$, we obtain that $\left(G(\mathbf{K}),\left[S_{n c}, R\right](\mathbf{K})\right)$ has relative Property (T). Since $\left(G(\mathbf{K}), S_{n h}(\mathbf{K})\right)$ also has relative Property $(\mathrm{T})$ and $\left[S_{n c}, R\right](\mathbf{K})$ is a normal subgroup, we obtain that $\left(G(\mathbf{K}), R_{T}(\mathbf{K})\right)$ has relative Property (T) by Proposition 2.4.1.

To show that $R_{T}$ is a $\mathbf{K}$-characteristic subgroup, we can work modulo the subgroup $\left[S_{n c}, R\right]$ which is K-characteristic by Lemma 3.2.2. But, in $G /\left[S_{n c}, R\right], S_{n c}$ is a direct factor and can be characterized as the biggest normal subgroup that is connected, semisimple, and $\mathbf{K}$-isotropic; and $S_{n h}$ is $\mathbf{K}$-characteristic in $S_{n c}$. It follows that $R_{T}$ is $\mathbf{K}$-characteristic.

\footnotetext{
${ }^{2}(n) m$ stands for (non-)amenable.
}

$4^{\text {e }}$ SÉRIE - TOME $39-2006-\mathrm{N}^{\circ} 2$ 
Finally, $H=G / R_{T}$ is almost the direct product of a semisimple group $H_{s}$ such that $H_{s}(\mathbf{K})$ is Haagerup, and its amenable radical $H_{m}$, such that $H_{m}(\mathbf{K})$ is amenable, hence Haagerup. So $H(\mathbf{K})$ is Haagerup, and contains $G(\mathbf{K}) / R_{T}(\mathbf{K})$ as a closed subgroup.

So we are in position to apply Lemma 3.1.1.

COROLlaRY 3.2.5. - Let $X$ be a subset of $G(\mathbf{K})$. Then $(G(\mathbf{K}), X)$ has relative Property $(\mathrm{T})$ if and only if the image of $X$ in $G(\mathbf{K}) / R_{T}(\mathbf{K})$ is relatively compact.

We retrieve a result of Wang (his statement is slightly different but equivalent to this one).

Corollary 3.2.6 (Wang). $-G(\mathbf{K})$ has Property (T) if and only if $S_{n h}\left[S_{n c}, R_{u}\right](\mathbf{K})$ is cocompact in $G(\mathbf{K})$.

Corollary 3.2.7 (See [12]). $-G(\mathbf{K})$ is Haagerup if and only if $S_{n h}=\left[S_{n c}, R\right]=1$.

\subsection{Relative Property (T) in connected Lie groups}

Let $G$ be a Lie group (connected, even if it is straightforward to generalize what follows to a Lie group with finitely many components), $R$ its radical, $S$ a Levi factor (not necessarily closed), decomposed as $S_{c} S_{n c}$ by separating compact and noncompact factors. Let $S_{n h}$ be the sum of all simple factors of $S_{n c}$ that have Property (T). Set $R_{T}=\overline{S_{n h}\left[S_{n c}, R\right]}$.

THEOREM 3.3.1. $-R_{T}$ is a characteristic subgroup of $G, G / R_{T}$ is Haagerup, and $\left(G, R_{T}\right)$ has relative Property $(\mathrm{T})$.

Proof. - The first statement can be proved in the same lines as in the algebraic case.

It is immediate that $G / R_{T}$ is locally isomorphic to a direct product $M \times S$ where $M$ is amenable and $S$ is semisimple with all simple factors locally isomorphic to $\mathrm{SO}(n, 1)$ or $\mathrm{SU}(n, 1)$. By [10, Chapter 4], $G / R_{T}$ is Haagerup.

Finally, let us show that $\left(G, R_{T}\right)$ has relative Property (T). First, note that we can reduce to the case when $G$ is simply connected. Indeed, let $p: \widetilde{G} \rightarrow G$ be the universal covering. Then $p(\widetilde{H})=H$, for $H=R, S_{n c}, S_{n h}$, where $\widetilde{H}$ is the analytic subgroup of $\widetilde{G}$ that lies over $H$. If the simply connected case is done, then $\left(\widetilde{G}, \widetilde{S_{n h}}\left[\widetilde{S_{n c}}, \widetilde{R}\right]\right)$ has relative Property (T). It follows that $\left(G, p\left(\widetilde{S_{n h}}\left[\widetilde{S_{n c}}, \widetilde{R}\right]\right)\right)$ also has relative Property (T), and the closure of $p\left(\widetilde{S_{n h}}\left[\widetilde{S_{n c}}, \widetilde{R}\right]\right)$ is equal to $R_{T}$.

Now suppose that $G$ is simply connected. Then the subgroup $S_{n c}\left[S_{n c}, R\right]$ is closed and isomorphic to $S_{n c} \ltimes\left[S_{n c}, R\right]$. Arguing as in the proof of Lemma 3.2.1 (using Example 3.1.8(2) instead of (1)), $\left(S_{n c} \ltimes\left[S_{n c}, R\right],\left[S_{n c}, R\right]\right)$ has relative Property (T). Hence $\left(G,\left[S_{n c}, R\right]\right)$ also has relative Property (T), and, as in the proof of Theorem 3.2.4, it implies that $\left(G, S_{n h}\left[S_{n c}, R\right]\right)$ has relative Property $(\mathrm{T})$.

So we are again in position to apply Lemma 3.1.1.

Corollary 3.3.2. - Let $X$ be a subset of $G$. Then $(G, X)$ has relative Property $(\mathrm{T})$ if and only if the image of $X$ in $G / R_{T}$ is relatively compact.

We also retrieve a result of Wang in the case of connected Lie groups.

COROLlary 3.3 .3 (Wang). - The connected Lie group G has Property (T) if and only if $\overline{S_{n h}\left[S_{n c}, R_{u}\right]}$ is cocompact in $G$. 
COROLlary 3.3.4 (See [10, Chapter 4]). - The connected Lie group $G$ is Haagerup if and only if ${ }^{3} S_{n h}=\left[S_{n c}, R\right]=1$.

Proof of Corollary 3.3.4. - The hypothesis implies that $S_{n h}$ and $W=\left[S_{n c}, R\right]$ are both relatively compact. So $S_{n h}=1$. Now, since $\left[S_{n c},\left[S_{n c}, R\right]\right]=\left[S_{n c}, R\right]$, we have $\left[S_{n c}, \bar{W}\right]=\bar{W}$. But, since $W$ is a compact, connected, and solvable Lie group, it is a torus; since $S_{n c}$ is connected, its action on $\bar{W}$ is necessarily trivial, so that $W \subset\left[S_{n c}, \bar{W}\right]=1$.

Remark 3.3.5. - If $G$ is a connected Lie group without the Haagerup Property, the existence of a noncompact closed subgroup with relative Property (T) was proved in [10], and later established by another method in [12], where the result was generalized to linear algebraic groups over local fields of characteristic zero. However, in both cases, the subgroup constructed is not necessarily normal.

Remark 3.3.6. - In this remark, given a locally compact group $G$, we say that a closed, normal subgroup $N$ is a T-radical if $G / N$ is Haagerup and $(G, N)$ has relative Property (T).

It is natural to ask about the uniqueness of T-radicals when they exist. Observe that if $N, N^{\prime}$ are T-radicals, then the image of $N$ in $G / N^{\prime}$ is relatively compact, and vice versa. In particular, if $G$ is discrete, then all T-radicals are commensurable. This is no longer the case if $G$ is not discrete, for instance, set $G=\mathrm{SL}(2, \mathbf{Z}) \ltimes \mathbf{R}^{2}$. Then the subgroups $a \mathbf{Z}^{2}$, for $a \neq 0$, are all T-radicals, although two of them may have trivial intersection.

In this example, $G$ has no minimal T-radical. This is also the case in $\operatorname{SL}(2, \mathbf{Z}) \ltimes \mathbf{Z}^{2}$. On the other hand, let $G$ be a finitely generated solvable group with infinite locally finite centre. Then, every finite subgroup of the centre is a T-radical, but $G$ has no infinite T-radical, so has no maximal T-radical. An example of such a group $G$ is the group of matrices of the form $\left(\begin{array}{ccc}1 & a & b \\ 0 & u^{n} & c \\ 0 & 0 & 1\end{array}\right)$, for $a, b, c \in \mathbf{Z}\left[u, u^{-1}\right], n \in \mathbf{Z}$.

However, if $G$ is a connected Lie group, it can be shown that $G$ has a minimal and a maximal T-radical. The minimal one is $R_{T}$, as defined above: indeed, if $H$ is a quotient of $G$ with the Haagerup Property, then $S_{n h}$ and $\left[S_{n c}, R\right]$ are necessarily contained in the kernel. The maximal one is found by taking the preimage of the maximal normal compact subgroup of $G / R_{T}$; it can immediately be generalized to any connected locally compact group.

Remark 3.3.7. - Following Shalom [36], if $G$ is a topological group and $H$ is a subgroup, we say that $(G, H)$ has strong relative Property (T) if there exists a Kazhdan pair $(K, \varepsilon)$ for the pair $(G, H)$ with $K$ finite (and $\varepsilon>0$ ). More precisely, this means that every unitary representation with a $(K, \varepsilon)$-invariant vector has a $H$-invariant vector. In this context, it is natural to equip $\widehat{G}$ with the topology inherited from $\widehat{G_{d}}$, the unitary dual of $G_{d}$, where $G_{d}$ denotes $G$ with the discrete topology. As for the case of relative Property (T), it can be checked that $(G, H)$ has strong relative Property (T) if and only if, for every net $\pi_{i}$ in $\widehat{G}$ converging to 1 in $\widehat{G_{d}}$, eventually $\pi_{i}$ has a $H$-invariant vector. Then it is straightforward from the proof that Proposition 3.1.3 remains true for strong relative Property (T). On the other hand, Proposition 3.1.2 is actually true with strong Property (T) [36, Theorem 5.5]. It then follows from the proofs above that, if $G$ is a connected Lie group, then $\left(G, R_{T}\right)$ has strong relative Property $(\mathrm{T})$, and similarly for algebraic groups over local fields of characteristic zero.

\footnotetext{
${ }^{3}$ Note that we used, in the proof of Theorem 3.3.1, one implication from [10, Chapter 4], namely, $S_{n h}=\left[S_{n c}, R\right]=1$ implies $G$ Haagerup. This result is easy when $S_{n c}$ has finite centre, but, otherwise, is much more involved. Accordingly, only the reverse implication can be considered as a corollary of the present work.

$4^{\mathrm{e}}$ SÉRIE - TOME $39-2006-\mathrm{N}^{\circ} 2$
} 


\section{Framework for irreducible lattices: resolutions}

In this section, we make a systematic study of ideas relying on work of Lubotzky and Zimmer [26], and later apparent in [29, Chapter III, 6] and [4].

Given a locally compact group $G$, when can we say that we have a good quantification of Kazhdan's Property (T)? Lemma 3.1.1 provides a satisfactory answer whenever $G$ has a normal subgroup $N$ such that $G / N$ is Haagerup and $(G, N)$ has relative Property (T). We have seen in Section 3 that this is satisfied in a large class of groups. However, this is not inherited by lattices. A typical example is the case of an irreducible lattice $\Gamma$ in a product of noncompact simple connected Lie groups $G \times H$, where $G$ has Property (T) and $H$ is Haagerup. In such an example, although $\Gamma \cap G=\{1\}, G$ can be thought as a "ghost" normal sugroup of $\Gamma$, and is the "kernel" of the projection $\Gamma \rightarrow H$. Relative Property (T) for the pair $(G \times H, G)$ can be restated by saying that the projection $G \times H \rightarrow H$ is a "resolution". By a theorem essentially due to Margulis (Theorem 4.3.1), this notion is inherited by lattices, so that, in this case, the projection $\Gamma \rightarrow H$ is a resolution.

Before giving rigorous definitions, we need some elementary preliminaries.

\section{1. $Q$-points}

We recall that an action $\alpha$ by isometries of a topological group $G$ on a metric space $X$ is continuous if the function $g \mapsto \alpha(g) x$ is continuous for every $x \in X$. All the functions and actions here are supposed continuous.

Let $f: G \rightarrow Q$ be a morphism between topological groups, with dense image. Recall that, for any Hausdorff topological space $X$, a function $u: G \rightarrow X$ factors through $Q$ if and only if, for every net $\left(g_{i}\right)$ in $G$ such that $f\left(g_{i}\right)$ converges in $Q$, the net $u\left(g_{i}\right)$ converges in $X$; note that the factorization $Q \rightarrow X$ is unique.

DEFINITION 4.1.1. - Let $f: G \rightarrow Q$ be a morphism between topological groups, with dense image. Let $\alpha$ be an action of $G$ by isometries on a metric space $X$. We call $x \in X$ a $Q$-point if the orbital map $g \mapsto \alpha(g) x$ factors through $Q$.

Proposition 4.1.2. - The set $X^{Q}$ of $Q$-points in $X$ is $G$-invariant, and the action $\alpha^{Q}$ of $G$ on $X^{Q}$ factors through $Q$. If moreover $X$ is a complete metric space, then $X^{Q}$ is closed in $X$.

Proof. - The first assertion is immediate; let us assume that $X$ is complete and let us show that $X^{Q}$ is closed. Let $\left(y_{n}\right)$ be a sequence in $X^{Q}$, converging to a point $y \in X$. Write $\alpha(g) y_{n}=$ $w_{n}(f(g))$, where $w_{n}$ is a continuous function: $Q \rightarrow X$. If $m, n \in \mathbf{N}, d\left(w_{m}(f(g)), w_{n}(f(g))\right)=$ $d\left(\alpha(g) y_{m}, \alpha(g) y_{n}\right)=d\left(y_{m}, y_{n}\right)$. It follows that $\sup _{q \in f(G)} d\left(w_{m}(q), w_{n}(q)\right) \rightarrow 0$ when $m, n \rightarrow \infty$. On the other hand, since $f(G)$ is dense in $Q, \sup _{q \in f(G)} d\left(w_{m}(q), w_{n}(q)\right)=$ $\sup _{q \in Q} d\left(w_{m}(q), w_{n}(q)\right)$. Accordingly, $\left(w_{n}\right)$ is a Cauchy sequence for the topology of uniform convergence on $Q$. Since $X$ is complete, this implies that $\left(w_{n}\right)$ converges to a continuous function $w: Q \rightarrow X$. Clearly, for all $g \in G, \alpha(g) y=w(f(g))$, so that $y \in X^{Q}$.

Proposition 4.1.3. - Suppose that $X$ is a complete metric space. Given $x \in X$, the following are equivalent:

(1) $x \in X^{Q}$.

(2) The mapping $g \mapsto d(x, \alpha(g) x)$ factors through $Q$.

(3) For every net $\left(g_{i}\right)$ in $G$ such that $f\left(g_{i}\right) \rightarrow 1, d\left(x, \alpha\left(g_{i}\right) x\right) \rightarrow 0$.

Proof. $-(1) \Rightarrow(2) \Rightarrow(3)$ is immediate. 
Suppose (3). Let $\left(g_{i}\right)$ be a net in $G$ such that $f\left(g_{i}\right)$ converges in $Q$. Then $\left(f\left(g_{i}^{-1} g_{j}\right)\right)$ converges to 1 when $i, j \rightarrow \infty$, so that

$$
d\left(\alpha\left(g_{i}\right) x, \alpha\left(g_{j}\right) x\right)=d\left(y, \alpha\left(g_{i}^{-1} g_{j}\right) x\right) \rightarrow 0
$$

i.e. $\left(\alpha\left(g_{i}\right) x\right)$ is Cauchy. Hence, it converges since $X$ is complete. This means that $g \mapsto \alpha(g) x$ factors through $Q$, i.e. $x \in X^{Q}$.

Recall that CAT $(0)$ metric spaces are a generalization of simply connected Riemannian manifolds with non-positive curvature; see [7] for a definition.

Proposition 4.1.4. -

(1) Suppose that $X$ is a complete $\mathrm{CAT}(0)$ metric space. Then $X^{Q}$ is a closed, totally geodesic subspace.

(2) If $X=\mathscr{H}$ is the Hilbert space of a unitary representation $\pi$ of $G$, then $\mathscr{H}^{Q}$ is a closed subspace, defining a subrepresentation $\pi^{Q}$ of $\pi$ (we refer to elements in $\mathscr{H}^{Q}$ as $Q$ vectors rather that $Q$-points). For every $\xi \in \mathscr{H}, \xi \in \mathscr{H}^{Q}$ if and only if the corresponding coefficient $g \mapsto\langle\xi, \pi(g) \xi\rangle$ factors through $Q$.

(3) If $X=\mathscr{H}$ is an affine Hilbert space, then $\mathscr{H}^{Q}$ is a closed affine subspace (possibly empty). For every $v \in \mathscr{H}, v \in \mathscr{H}^{Q}$ if and only if the corresponding conditionally negative definite function $g \mapsto\|v-g \cdot v\|^{2}$ factors through $Q$.

Proof. - (1) The first statement is immediate since, for all $\lambda \in \mathbf{R}$, the function $\left(c, c^{\prime}\right) \mapsto$ $(1-\lambda) c+\lambda c^{\prime}$ is continuous (actually 1-Lipschitz) on its domain of definition.

(2) If $\mathscr{H}$ is the Hilbert space of a unitary representation, then $\mathscr{H}^{Q}$ is immediately seen to be a linear subspace, and is closed by Proposition 4.1.2. Note that this also can be derived as a particular case of (1). The nontrivial part of the last statement in (2) follows from Proposition 4.1.3.

(3) is similar.

LEMMA 4.1.5. - Let $G \rightarrow Q$ be a morphism with dense image, and $\left(\pi_{i}\right)$ is a family of unitary representations of $G$. Then $\left(\bigoplus \pi_{i}\right)^{Q}=\bigoplus \pi_{i}^{Q}$.

Proof. - The inclusion $\bigoplus \pi_{i}^{Q} \subset\left(\bigoplus \pi_{i}\right)^{Q}$ is trivial. Let $p_{i}$ denote the natural projections, set $\pi=\bigoplus \pi_{i}$, and write $\mu_{\xi}(g)=\pi(g) \xi$. Then, if $\xi$ is a $Q$-vector, i.e. if $\mu_{\xi}$ factors through $Q$, then $p_{i} \circ \mu_{\xi}$ also factors through $Q$. But $p_{i} \circ \mu_{\xi}=\mu_{p_{i}(\xi)}$, so that $p_{i}(\xi)$ is a $Q$-vector.

\subsection{Resolutions}

Convention 4.2.1. - If $G \rightarrow Q$ is a morphism with dense image, and $\pi$ is a unitary representation of $G$ factoring through a representation $\tilde{\pi}$ of $Q$, we write $1_{Q} \prec \pi$ rather than $1_{Q} \prec \tilde{\pi}$ or $1 \prec \tilde{\pi}$ to say that $\tilde{\pi}$ almost has invariant vectors (note that $1_{Q} \prec \pi$ implies $1_{G} \prec \pi$, but the converse is not true in general). Similarly, if $\left(\pi_{i}\right)$ is a net of unitary representations of $G$ factoring through representations $\tilde{\pi}_{i}$ of $Q$, when we write $\pi_{i} \rightarrow 1_{Q}$, we mean for the Fell topology on unitary representations of $Q$.

DEFINITION 4.2.2 (Resolutions). - Let $G$ be a locally compact group, and $f: G \rightarrow Q$ a morphism to another locally compact group $Q$, such that $f(G)$ is dense in $Q$.

We say that $f$ is a resolution if, for every unitary representation $\pi$ of $G$ almost having invariant vectors, then $1_{Q} \prec \pi^{Q}$, meaning that $\pi^{Q}$, viewed as a representation of $Q$, almost has invariant vectors (in particular, $\pi^{Q} \neq 0$ ).

We call $f$ a Haagerup resolution if $Q$ is Haagerup.

$4^{\text {e }}$ SÉRIE - TOME $39-2006-\mathrm{N}^{\circ} 2$ 
The definition of resolution generalizes the notion of relative Property $(\mathrm{T})$ of a closed normal subgroup $N$ of $G$, since $G \rightarrow G / N$ is a resolution if and only if $(G, N)$ has relative Property (T).

Remark 4.2.3. - When $G$ is $\sigma$-compact, it can be shown $[14, \S 2.3 .8]$ that a morphism $f: G \rightarrow Q$ is a resolution if and only if it satisfies the (a priori weaker) condition: every unitary representation $\pi$ of $G$ such that $1_{G} \prec \pi$ satisfies $\pi^{Q} \neq 0$. The proof is not immediate and makes use of the results in the sequel (namely, Theorem 4.7.6).

PROPOSITION 4.2.4. - Let $f: G \rightarrow Q$ be a morphism between locally compact groups, with dense image. The following are equivalent:

(1) $f$ is a resolution.

(2) For every net $\left(\pi_{i}\right)$ of unitary representations of $G$ converging to $1_{G}$, we have $\pi_{i}^{Q} \rightarrow 1_{Q}$.

Proof. - (2) $\Rightarrow$ (1) is trivial. Suppose (1). Let $\pi_{i} \rightarrow 1_{G}$. Then, for every subnet $\left(\pi_{j}\right), 1_{G} \prec$ $\bigoplus_{j} \pi_{j}$. By (1), $1_{Q} \prec\left(\bigoplus_{j} \pi_{j}\right)^{Q}$, which equals $\bigoplus_{j} \pi_{j}^{Q}$ by Lemma 4.1.5. Hence $\pi_{i}^{Q} \rightarrow 1_{Q}$.

COROLlaRY 4.2.5. - Let $G \rightarrow Q$ be a resolution. Then for every net $\left(\pi_{i}\right)$ of irreducible unitary representations of $G$ converging to $1_{G}$, eventually $\pi_{i}$ factors through a representation $\tilde{\pi}_{i}$ of $Q$, and $\tilde{\pi}_{i} \rightarrow 1_{Q}$.

The converse of Corollary 4.2.5 is more involved, and is proved (Theorem 4.7.11) under the mild hypothesis that $G$ is $\sigma$-compact.

Thus, a resolution allows to convey properties about the neighbourhood of $1_{Q}$ in $\widehat{Q}$ into properties about the neighbourhood of $1_{G}$ in $\widehat{G}$. For instance, this is illustrated by Property $(\tau)$ (see Section 4.5).

The following proposition generalizes the fact that relative Property $(T)$ is inherited by extensions (Proposition 2.4.4), and is one of our main motivations for having introduced resolutions.

Proposition 4.2.6. - Let $f: G \rightarrow Q$ be a resolution, and $X \subset G$. Then $(G, X)$ has relative Property (T) if and only if $(Q, f(X))$ does so.

Proof. - The condition is trivially sufficient. Suppose that $(Q, f(X))$ has relative Property (T). Fix $\varepsilon>0$, and let $\pi$ be a unitary representation of $G$ such that $1_{G} \prec \pi$. Since $G \rightarrow Q$ is a resolution, $1_{Q} \prec \pi^{Q}$. Hence, by Property (T), $\pi^{Q}$ has a $(f(X), \varepsilon)$-invariant vector; this is a $(X, \varepsilon)$-invariant vector for $\pi$.

Recall that a morphism between locally compact spaces is proper if the inverse image of any compact subset is compact. It is easy to check that a morphism $G \rightarrow H$ between locally compact groups is proper if and only if its kernel $K$ is compact, its image $Q$ is closed in $H$, and the induced map $G / K \rightarrow Q$ is an isomorphism of topological groups.

COROLlary 4.2.7. - Let $f: G \rightarrow Q$ be a Haagerup resolution. Then for every $X \subset G$, $(G, X)$ has relative Property $(\mathrm{T})$ if and only if $\overline{f(X)}$ is compact.

Accordingly, either $f$ is a proper morphism, so that $G$ is also Haagerup, or there exists a noncompact closed subset $X \subset G$ such that $(G, X)$ has relative Property (T). In particular, $G$ satisfies the TH alternative.

THEOREM 4.2.8. - Let $f: G \rightarrow Q$ be a resolution. Then $G$ is compactly generated if and only if $Q$ is so.

This theorem generalizes compact generation of locally compact groups with Property (T) [24] (case when $Q=\{1\}$ ); in this more specific direction, it generalizes Proposition 2.8 of [26]. 
LEMMA 4.2.9. - Let $f: G \rightarrow Q$ be a morphism with dense image between topological groups, and let $\Omega$ be an open neighbourhood of 1 of $Q$. Then, for all $n, f^{-1}\left(\Omega^{n}\right) \subset f^{-1}(\Omega)^{n+1}$.

Proof. - Let $x$ belong to $f^{-1}\left(\Omega^{n}\right)$. Write $f(x)=u_{1} \cdots u_{n}$ with $u_{i} \in \Omega$. Consider $\left(\varepsilon_{1}, \ldots, \varepsilon_{n}\right) \in \Omega^{n}$. Set $v_{i}=u_{i} \varepsilon_{i}$ and $v_{0}=u_{1} \cdots u_{n}\left(v_{1} \cdots v_{n}\right)^{-1}$, so that $f(x)=v_{0} v_{1} \cdots v_{n}$. If all $\varepsilon_{i}$ are chosen sufficiently close to 1 , then $v_{0} \in \Omega$ and $u_{i} \varepsilon_{i} \in \Omega$ for all $i$; by density of $f(G)$, we can also impose that $u_{i} \varepsilon_{i} \in f(G)$ for all $i$. We fix $\varepsilon_{1}, \ldots, \varepsilon_{n}$ so that all these conditions are satisfied. Since $v_{0}=f(x)\left(v_{1} \cdots v_{n}\right)^{-1}$, we observe that $v_{0}$ also belongs to $f(G)$. For all $i$, write $v_{i}=f\left(x_{i}\right)$, so that $x=k x_{0} x_{1} \cdots x_{n}$ with $k \in \operatorname{Ker}(f)$. Set $y_{0}=k x_{0}$. Then $x=y_{0} x_{1} \cdots x_{n} \in f^{-1}(\Omega)^{n+1}$.

Proof of Theorem 4.2.8. - If $G$ is compactly generated, so is $Q$ (only supposing that the morphism has dense image). Indeed, if $K$ is a compact generating set of $G$, then $f(K)$ generates a dense subgroup of $Q$. It follows that, if $K^{\prime}$ is a compact subset of $Q$ containing $f(K)$ in its interior, then $K^{\prime}$ generates $Q$.

Conversely, suppose that $Q$ is compactly generated, and let $\Omega$ be an open, relatively compact generating set. For every open, compactly generated subgroup $H$ of $G$, let $\varphi_{H}$ be its characteristic function. Then, when $H$ becomes big, $\varphi_{H}$ converges to 1 , uniformly on compact subsets of $G$. Since $\left(G, f^{-1}(\Omega)\right)$ has relative Property (T) by Proposition 4.2.6, it follows from Lemma 2.5.1 that $f^{-1}(\Omega)$ is contained in a compactly generated subgroup $H$ of $G$. By Lemma 4.2.9, $f^{-1}\left(\Omega^{n}\right) \subset f^{-1}(\Omega)^{n+1} \subset H$. Since $Q=\bigcup \Omega^{n}$, it follows that $G=H$.

\subsection{Lattices and resolutions}

The following theorem generalizes the fact that Property $(\mathrm{T})$ is inherited by lattices.

THEOREM 4.3.1. - Let $G$ be a locally compact group, $N$ a closed, normal subgroup. Suppose that $(G, N)$ has relative Property (T) (equivalently, the projection $f: G \rightarrow G / N$ is a resolution).

Let $H$ be a closed subgroup of finite covolume in $G$, and write $Q=\overline{f(H)}$. Then $f: H \rightarrow Q$ is a resolution.

Theorem 4.3.1 is a slight strengthening of [29, Chapter III, (6.3) Theorem]. To prove it, we need the following lemma, whose ingredients are borrowed from [4].

LEMma 4.3.2. - Let $G, N, H, Q$ be as in Theorem 4.3.1. For every unitary representation $\pi$ of $H$ factoring through $Q$, if $1_{H} \prec \pi$, then $1_{Q} \prec \pi$.

Proof. - By [23, Corollary 4.1(2)], $\left(f^{-1}(Q), N\right)$ has relative Property (T); hence, replacing $G$ by $f^{-1}(Q)$ if necessary, we can suppose that $Q=G / N$.

Denote by $\tilde{\pi}$ the factorization of $\pi$ through $Q$, and by $\hat{\pi}$ the (intermediate) factorization of $\pi$ through $G$, so that $\pi=\left.\hat{\pi}\right|_{H}$.

By continuity of induction and since $H$ has finite covolume, $1_{G} \prec \operatorname{Ind}_{H}^{G} \pi$. On the other hand, $\operatorname{Ind}_{H}^{G} \pi=\left.\operatorname{Ind}_{H}^{G} \hat{\pi}\right|_{H}=\hat{\pi} \otimes L^{2}(G / H)=\hat{\pi} \oplus\left(\hat{\pi} \otimes L_{0}^{2}(G / H)\right)$.

We claim that $1_{G} \nprec \hat{\pi} \otimes L_{0}^{2}(G / H)$. It follows that $1_{G} \prec \hat{\pi}$. Since every compact subset of $G / N$ is the image of a compact subset of $G$ [3, Lemma B.1.1], it follows that $1_{G / N} \prec \tilde{\pi}$.

It remains to prove the claim. Since $\left.\hat{\pi}\right|_{N}$ is a trivial representation, $\left.\left.\hat{\pi}\right|_{N} \otimes L_{0}^{2}(G / H)\right|_{N}$ is a multiple of $\left.L_{0}^{2}(G / H)\right|_{N}$. But, by [4, Lemma 2], $L_{0}^{2}(G / H)$ does not contain any nonzero $N$-invariant vector. Accordingly, neither does $\hat{\pi} \otimes L_{0}^{2}(G / H)$. Hence, by relative Property (T), $1_{G} \nprec \hat{\pi} \otimes L_{0}^{2}(G / H)$.

$4^{\text {e }}$ SÉRIE - TOME $39-2006-\mathrm{N}^{\circ} 2$ 
Proof of Theorem 4.3.1. - Let $\pi$ be a unitary representation of $H$, and suppose that $1_{H} \prec \pi$. Using [29, Chapter III, (6.3) Theorem] twice ${ }^{4}, \pi^{Q} \neq 0$, and its orthogonal in $\pi$ does not almost contain invariant vectors. It follows that $1_{H} \prec \pi^{Q}$. By Lemma 4.3.2, $1_{Q} \prec \pi^{Q}$.

Remark 4.3.3. - The conclusion of Lemma 4.3.2 is false if we drop the assumption that $(G, N)$ has relative Property $(\mathrm{T})$, as the following example shows.

Set $G=\mathbf{Z} \times \mathbf{R} / \mathbf{Z}$ and $N=\mathbf{Z} \times\{0\}$. Let $H$ be the cyclic subgroup of $G$ generated by $(1, \alpha)$, where $\alpha \in(\mathbf{R}-\mathbf{Q}) / \mathbf{Z}$.

The projection $p: H \rightarrow \mathbf{R} / \mathbf{Z}$ has dense image. Hence, the Pontryagin dual morphism: $\hat{p}: \mathbf{Z} \simeq$ $\widehat{\mathbf{R} / \mathbf{Z}} \rightarrow H^{*} \simeq \mathbf{R} / \mathbf{Z}$ also has dense image. Take a sequence $\left(\chi_{n}\right)$ of pairwise distinct nontrivial characters of $\mathbf{R} / \mathbf{Z}$ such that $\hat{p}\left(\chi_{n}\right)$ tends to 0 . Then the direct sum $\pi=\bigoplus \chi_{n}$ does not weakly contain the trivial representation (otherwise, since $\mathbf{R} / \mathbf{Z}$ has Property (T), it would contain the trivial representation), but $\left.\pi \circ p\right|_{H}$ weakly contains the trivial representation $1_{H}$.

We can now combine the results of Section 3 with Theorem 4.3.1. Let $G$ be a finite direct product of connected Lie groups and algebraic groups over local fields of characteristic zero: $G=L \times \prod_{i=1}^{n} H_{i}\left(\mathbf{K}_{i}\right)$. Write $R_{T}(G)=R_{T}(L) \times \prod_{i=1}^{n} R_{T}\left(H_{i}\right)\left(\mathbf{K}_{i}\right)$, where $R_{T}$ is defined in Sections 3.2 and 3.3. Observe that, by Theorems 3.3.1 and 3.2.4, $\left(G, R_{T}\right)$ has relative Property (T) and $G / R_{T}$ is Haagerup. Denote by $f: G \rightarrow G / R_{T}(G)$ the quotient morphism.

COROLLARY 4.3.4. - Let $G$ be a finite product of connected Lie groups and (rational points of) algebraic groups over local fields of characteristic zero. Let $\Gamma$ be a closed subgroup of finite covolume in $G$. Then there exists a Haagerup resolution for $\Gamma$, given by $f: \Gamma \rightarrow \overline{f(\Gamma)}$.

\subsection{The factorization theorem}

THEOREM 4.4.1. - Let $G$ be a locally compact group, $f: G \rightarrow Q$ a resolution, $u: G \rightarrow H$ a morphism to a locally compact group, with dense image, where $H$ is Haagerup. Then there exist a compact, normal subgroup $K$ of $H$, and a factorization $Q \rightarrow H / K$ making the following diagram commutative

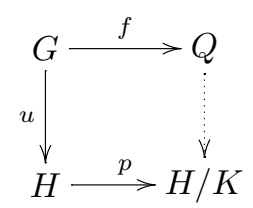

Proof. - $H$ has a $C_{0}$ unitary representation $\pi$ with almost invariant vectors. Therefore $\pi \circ u$ almost has invariant vectors, so that, passing to a subrepresentation if necessary, we can suppose that $\pi \circ u$ factors through a representation $\tilde{\pi}$ of $Q$. We fix a normalized coefficient $\varphi$ of $\tilde{\pi} \circ f$.

Let $\left(g_{i}\right)$ be a net in $G$ such that $f\left(g_{i}\right) \rightarrow 1$. Then $\varphi\left(g_{i}\right) \rightarrow 1$. This implies that $\left(u\left(g_{i}\right)\right)$ is bounded in $H$, since $\varphi$ is a $C_{0}$ function. Let $K \subset H$ be the set of all limits of $u\left(g_{i}\right)$ for such nets $\left(g_{i}\right)$. Then $K$ is a compact, normal subgroup of $H$ (it is normal thanks to the density of $u(G)$ in $H$ ).

Let $p$ be the projection: $H \rightarrow H / K$. We claim that $p \circ u$ factors through $Q$. Indeed, if $\left(g_{i}\right)$ is a net in $G$ such that $\left(f\left(g_{i}\right)\right)$ is Cauchy in $Q$, then $p \circ u$ is also Cauchy in $H / K$. This implies that $p \circ u$ factors through $Q$.

\footnotetext{
${ }^{4}$ The assumption in [29] is that $N$ has Property (T), but it is clear from the proof that relative Property (T) for $(G, N)$ is sufficient.
} 


\subsection{Applications to Property $(\tau)$ and related properties}

Recall that a representation of a group is said to be finite if its kernel has finite index.

DEFINITION 4.5.1. - We recall that a topological group $G$ has Property $(\tau)$ [respectively $\left.\left(\tau_{F D}\right)\right]$ if for every net $\left(\pi_{i}\right)$ of finite (respectively finite-dimensional) irreducible unitary representations of $G$ converging to $1_{G}$, eventually $\pi_{i}=1_{G}$.

We say that a topological group $G$ has Property $\left(\mathrm{FH}_{F D}\right)$ if every isometric action of $G$ on a finite-dimensional Hilbert space has a fixed point. Equivalently, every finite-dimensional unitary representation has vanishing 1-cohomology.

We say that a topological group $G$ has Property $\left(\mathrm{FH}_{F}\right)$ if every finite unitary representation has vanishing 1-cohomology.

The topological group $G$ has Property $\left(\mathrm{FAb}^{\mathbf{R}}\right)$ [respectively (FAb)] if for every closed subgroup of finite index $H$ of $G$, we have $\operatorname{Hom}(H, \mathbf{R})=0$ [respectively $\operatorname{Hom}(H, \mathbf{Z})=0$ ].

It turns out that Properties $\left(\mathrm{FH}_{F}\right)$ and $\left(\mathrm{FAb}^{\mathbf{R}}\right)$ are equivalent. This is shown in [27] ${ }^{5}$ using induction of unitary representations and Shapiro's Lemma. Alternatively, this can be shown using induction of affine representations.

Note also that $\left(\mathrm{FAb}^{\mathbf{R}}\right)$ implies $(\mathrm{FAb})$, and they are clearly equivalent for finitely generated groups; while $\mathbf{R}$ or $\mathbf{Q}$ satisfy (FAb) but not $\left(\mathrm{FAb}^{\mathbf{R}}\right)$.

Property $(\tau)$ clearly implies (FAb) [27]; it is there observed that the first Grigorchuk group does not have Property $(\tau)$, but has Property $\left(\mathrm{FH}_{F D}\right)$ since all its linear representations are finite. However, no finitely presented group is known to satisfy (FAb) but not Property $(\tau)$.

We provide below an example of a finitely generated group having Property $(\tau)$ (hence $\left(\mathrm{FH}_{F}\right)$ ) but not $\left(\mathrm{FH}_{F D}\right)$. We do not know if this is the first known example.

We begin by a general result.

Proposition 4.5.2. - Let $G, Q$ be locally compact, and $G \rightarrow Q$ be a resolution. Let $(\mathrm{P})$ be one of the properties: $(\mathrm{T}),(\tau),\left(\tau_{F D}\right),(\mathrm{FAb}),\left(\mathrm{FAb}^{\mathbf{R}}\right),\left(\mathrm{FH}_{F}\right),\left(\mathrm{FH}_{F D}\right)$. Then $G$ has Property $(\mathrm{P})$ if and only if $Q$ does so.

Proof. - In all cases, Property (P) for $G$ clearly implies Property (P) for $Q$.

Let us show the converse. For (T), $(\tau)$, and $\left(\tau_{F D}\right)$ this follows directly from Proposition 4.2.4.

Suppose that $G$ does not have Property (FAb). Let $N \subset G$ be a closed normal subgroup of finite index such that $\operatorname{Hom}(N, \mathbf{Z}) \neq 0$. Let $M$ be the kernel of a morphism of $N$ onto $\mathbf{Z}$, and set $K=\bigcap_{g \in G / N} g M g^{-1}$. Then $K$ is the kernel of the natural diagonal morphism $N \rightarrow \prod_{g \in G / N} N / g M g^{-1} \simeq \mathbf{Z}^{G / N}$. It follows that $N / K$ is a nontrivial free abelian group of finite rank, and $K$ is normal in $G$, so that $H=G / K$ is infinite, finitely generated, virtually abelian. Since $H$ is Haagerup, by Theorem 4.4.1, $Q$ maps onto the quotient of $H$ by a finite subgroup $F$. Since $H / F$ is also infinite, finitely generated, virtually abelian, $Q$ does not have Property (FAb).

The case of Property $\left(\mathrm{FAb}^{\mathbf{R}}\right)$ can be proved similarly; since $\left(\mathrm{FAb}^{\mathbf{R}}\right)$ is equivalent to $\left(\mathrm{FH}_{F}\right)$ which is treated below, we omit the details.

Suppose that $G$ does not have Property $\left(\mathrm{FH}_{F D}\right)$. Let $G$ act isometrically on a Euclidean space $E$ with unbounded orbits, defining a morphism $\alpha: G \rightarrow \operatorname{Isom}(E)$. Set $H=\overline{\alpha(G)}$. Since $\operatorname{Isom}(E)$ is Haagerup, so is $H$. By Theorem 4.4.1, $H$ has a compact normal subgroup $K$ such that $Q$ has a morphism with dense image into $H / K$. Observe that the set of $K$-fixed points provides an action of $H / K$ on a nonempty affine subspace of $E$, with unbounded orbits. So $Q$ does not have Property $\left(\mathrm{FH}_{F D}\right)$.

\footnotetext{
${ }^{5}$ The group there is assumed to be finitely generated but this has no importance.

$4^{\mathrm{e}}$ SÉRIE - TOME $39-2006-\mathrm{N}^{\circ} 2$
} 
The case of Property $\left(\mathrm{FH}_{F}\right)$ can be treated similarly, noting that $\alpha$ maps a subgroup of finite index to translations, and this is preserved after restricting to the action on an affine subspace.

Remark 4.5.3. - The case of Property $\left(\mathrm{FH}_{F D}\right)$ in Proposition 4.5.2 contains as a particular case Theorem B in [4], without making use of the Vershik-Karpushev Theorem, while the proof given in [4] does.

Proposition 4.5.2 justifies why we do not have restricted the definitions of Property $(\tau)$, etc., to discrete groups (as is usually done), since in many cases, when we have a resolution $G \rightarrow Q$, the group $Q$ is non-discrete. For instance, all these properties are easy or trivial to characterize for connected Lie groups.

PROPOSITION 4.5.4. - Let $G$ be a connected locally compact group. Then

(1) G has Property $(\tau)$.

(2) $G$ has Property $\left(\mathrm{FH}_{F}\right)$ if and only if $\operatorname{Hom}(G, \mathbf{R})=0$.

(3) $G$ has Property $\left(\tau_{F D}\right)$ if and only if $\operatorname{Hom}(G, \mathbf{R})=0$.

(4) G has Property $\left(\mathrm{FH}_{F D}\right)$ if and only if every amenable quotient of $G$ is compact.

Proof. - Since $G$ has no proper closed finite index subgroup, (1) and (2) are immediate.

(3) The condition is clearly necessary. Conversely, suppose that $\operatorname{Hom}(G, \mathbf{R})=0$. Let $W$ be the intersection of all kernels of finite-dimensional unitary representations of $G$. Clearly, it suffices to show that $G / W$ has Property $\left(\tau_{F D}\right)$. By [17, Théorème 16.4.6], $G / W \simeq \mathbf{R}^{n} \times K$ for some compact group $K$. The assumption then implies $n=0$, so that $G / W$ is compact, so that $G$ has Property $\left(\tau_{F D}\right)$.

(4) Suppose that $G$ does not have Property $\left(\mathrm{FH}_{F D}\right)$. Then there exists an unbounded isometric affine action of $G$ on some Euclidean space. Let $K$ be a compact normal subgroup of $G$ such that $G / K$ is a Lie group [31]. Restricting to the (nonempty) affine subspace of $K$-fixed points, we can suppose that $K$ is contained in the kernel $N$ of this action. Necessarily, the Lie group $G / N$ is not compact, and amenable since it embeds in the amenable Lie group $\mathrm{O}(n) \ltimes \mathbf{R}^{n}$.

Conversely, suppose $G$ has a noncompact amenable quotient $H$. Since $H$ does not have Property (T), by a result of Shalom [37] (see [3, Section 3.2]), there exists an irreducible unitary representation $\pi$ of $H$ with non-vanishing 1-reduced cohomology. By [30, Theorem 3.1], $\pi$ is finite-dimensional. ${ }^{6}$

Proposition 4.5.5. - Fix $n \geqslant 5$, set $\Gamma=\mathrm{SO}_{n}\left(\mathbf{Z}\left[2^{1 / 3}\right]\right) \ltimes \mathbf{Z}\left[2^{1 / 3}\right]^{n}$. Then $\Gamma$ is a finitely presentable group, has Property $\left(\tau_{F D}\right)$ (hence Property $(\tau)$, hence Property $\left(\mathrm{FH}_{F}\right)$ ), but not $\left(\mathrm{FH}_{F D}\right)$.

Proof. - Note that $\mathrm{SO}_{n}(\mathbf{C})$ and $\mathrm{SO}_{n}(\mathbf{C}) \ltimes \mathbf{C}^{n}$ have Property (T). Since $\Gamma$ is an irreducible lattice in the connected Lie group $\left(\mathrm{SO}_{n}(\mathbf{R}) \ltimes \mathbf{R}^{n}\right) \times\left(\mathrm{SO}_{n}(\mathbf{C}) \ltimes \mathbf{C}^{n}\right)$, it is finitely presentable, and, moreover, by Theorem 4.3.1, $\Gamma \rightarrow \mathrm{SO}_{n}(\mathbf{R}) \ltimes \mathbf{R}^{n}$ is a resolution.

By Proposition 4.5.4, $\mathrm{SO}_{n}(\mathbf{R}) \ltimes \mathbf{R}^{n}$ has Property $\left(\tau_{F D}\right)$. Thus $\Gamma$ also has Property $\left(\tau_{F D}\right)$ by Proposition 4.5.2. On the other hand, the embedding of $\Gamma$ in $\mathrm{SO}_{n}(\mathbf{R}) \ltimes \mathbf{R}^{n}$ provides an isometric action of $\Gamma$ with unbounded orbits on the $n$-dimensional Euclidean space.

Remark 4.5.6. - It is asked in [26] whether there exists a finitely generated group with Property $(\tau)$ but not $\left(\tau_{F D}\right)$. Obvious non-finitely generated examples are $\mathbf{Q}$ and $\mathbf{R}$. It may be tempting to find a finitely generated group $\Gamma$ with a resolution $\Gamma \rightarrow \mathbf{R}$, but unfortunately no such $\Gamma$ exists. Indeed, since $\Gamma$ is discrete and $\operatorname{Hom}(\Gamma, \mathbf{R}) \neq 0$, there exists a discrete, nontrivial, torsion-free abelian quotient $\Lambda$ of $\Gamma$. By Theorem 4.4.1, there exists a factorization: $\mathbf{R} \rightarrow \Lambda$,

${ }^{6}$ It is possible to prove (4) more directly, but we have used Shalom's and Martin's results to make short. 
necessarily surjective. This is a contradiction since $\mathbf{R}$ is connected. Thus the question remains open.

\subsection{Subgroups of simple Lie groups}

Let $G$ be a connected simple Lie group, with Lie algebra $\mathfrak{g}$. We are interested in subgroups $\Gamma \subset G$, viewed as discrete groups.

(1) If $\mathfrak{g}$ is isomorphic to $\mathfrak{s l}_{2}(\mathbf{R}) \simeq \mathfrak{s o}(2,1) \simeq \mathfrak{s u}(1,1), \mathfrak{s l}_{2}(\mathbf{C}) \simeq \mathfrak{s o}(3,1)$, or $\mathfrak{s o}_{3}(\mathbf{R})$, then every $\Gamma \subset G$ is Haagerup [21, §5, Theorem 4] (see [12] for the case of $\widehat{\mathrm{SL}_{2}(\mathbf{R}) \text { ). }}$.

(2) If $G$ has Property (T) and $\mathfrak{g} \not \mathfrak{s o}_{3}(\mathbf{R})$, then there exists $\Gamma \subset G$ with Property (T): if $G$ is noncompact, take any lattice. If $G$ is compact, this is due to Margulis [29, Chapter III, Proposition 5.7].

(3) If $\mathfrak{g} \simeq \mathfrak{s o}(n, 1)$ with $n \geqslant 5$ or $\mathfrak{g} \simeq \mathfrak{s u}(n, 1)$ with $n \geqslant 3$, then there exists an infinite subgroup $\Gamma \subset G$ with Property (T): it suffices to observe that $G$ contains a subgroup locally isomorphic to $\mathrm{SO}(n)(n \geqslant 5)$ or $\mathrm{SU}(n)(n \geqslant 3)$, and such a subgroup contains an infinite subgroup with Property (T) by (2).

(4) There are only two remaining cases: $\mathfrak{g} \simeq \mathfrak{s o}(4,1)$ and $\mathfrak{g} \simeq \mathfrak{s u}(2,1)$. We are going to show that the behaviour there is different from that in preceding examples.

The only result already known is that if $\mathfrak{g} \simeq \mathfrak{s o}(4,1)$ or $\mathfrak{g} \simeq \mathfrak{s u}(2,1)$, then no infinite $\Gamma \subset G$ can have Property ( $\mathrm{T}$ ); this follows from (1) since such $\Gamma$ would be contained in a maximal compact subgroup. This result is generalized in the following theorem.

THEOREM 4.6.1. - Let $G$ be a connected Lie group, locally isomorphic to either $\mathrm{SO}(4,1)$ or $\mathrm{SU}(2,1)$. Let $\Gamma \subset G$ be any subgroup, and view $\Gamma$ as a discrete group.

(1) If $\Lambda \subset \Gamma$ is a normal subgroup such that $(\Gamma, \Lambda)$ has relative Property $(\mathrm{T})$, then $\Lambda$ is a finite subgroup of $G$.

(2) If $\Gamma$ is not dense, and if $\Lambda \subset \Gamma$ is a subgroup such that $(\Gamma, \Lambda)$ has relative Property $(\mathrm{T})$, then $\Lambda$ is a finite subgroup of $G$.

(3) If $\Gamma$ is dense, and $X \subset \Gamma$ is a normal subset (i.e. invariant under conjugation) such that $(\Gamma, X)$ has relative Property $(\mathrm{T})$, then $X$ is a finite subset of the centre of $G$.

Suppose that $G$ is locally isomorphic to $\mathrm{SU}(2,1)$. Then we have stronger statements:

(4) If $\Gamma$ is not dense, then $\Gamma$ is Haagerup.

(5) If $X \subset \Gamma$ is a normal subset and $(\Gamma, X)$ has relative Property ( $\mathrm{T})$, then $X$ is a finite subset of $G$.

Proof. - Fix a subset $X \subset \Gamma$ such that $(\Gamma, X)$ has relative Property (T). We make a series of observations.

(a) First note that $G$ is Haagerup, a fact due to [18] (and [10, Chapter 4] in the case of $\widetilde{\mathrm{SU}}(2,1))$. Therefore, by relative Property $(\mathrm{T}), \bar{X}$ must compact. Denote by $\mathfrak{h}$ the Lie algebra of $\bar{\Gamma}$.

(b) Suppose, in this paragraph (b), that $\Gamma$ is dense in $G$, i.e. $\mathfrak{h}=\mathfrak{g}$; and suppose that $X$ is a normal subset. Then $\bar{X}$ is a compact, normal subset in $G$. Let $Z$ be the centre of $G$, and fix $h \in X$. Then the conjugacy class of $h$ in $G / Z$ is relatively compact. Let $M$ be the symmetric space associated to $G / Z$, and fix $y \in M$. Then the function $g \mapsto d\left(g h g^{-1} y, y\right)$ is bounded, so that $h$ has bounded displacement length. Since $M$ is CAT(-1) and geodesically complete, this implies that $h$ acts as the identity, i.e. $h \in Z$. Accordingly, $X \subset Z$, so that $X$ is discrete. Since it is relatively compact, it is finite. This proves (3).

(c) Suppose that $\Gamma$ is Zariski dense modulo $Z$, but not dense. Then $\Gamma$ is discrete. Indeed, $\Gamma$ is contained in the stabilizer $W$ of $\mathfrak{h}$ for the adjoint action. Since $W$ is Zariski closed modulo $Z$,

$4^{\mathrm{e}}$ SÉRIE - TOME $39-2006-\mathrm{N}^{\circ} 2$ 
this implies that $\mathfrak{h}$ is an ideal in $\mathfrak{g}$, so that, since $\mathfrak{h} \neq \mathfrak{g}$ and $\mathfrak{g}$ is simple, $\mathfrak{h}=\{0\}$, i.e. $\Gamma$ is discrete. Since $G$ is Haagerup, this implies that $\Gamma$ is Haagerup.

(d) Now suppose that $Z=1$ and $\Gamma$ is not Zariski dense. Let $N$ be the Zariski closure of $\Gamma$. Let $R_{u}$ be the unipotent radical of $N$, and $L=C S$ a Levi factor, with abelian part $C$, and semisimple part $S$. The possibilities for simple factors in $S$ are rather restricted. The complexification $G_{\mathbf{C}}$ is isomorphic to either $\mathrm{PSO}_{5}(\mathbf{C})$ or $\mathrm{PSL}_{3}(\mathbf{C})$. In both cases, by a dimension argument, the only possible simple subgroups of $G_{\mathbf{C}}$ are, up to isogeny, $\mathrm{SL}_{2}(\mathbf{C})$, and maybe $\mathrm{SL}_{3}(\mathbf{C})$ in $\mathrm{PSO}_{5}(\mathbf{C})$; however, $\mathfrak{s l}_{3}(\mathbf{C})$ does not embed in $\mathfrak{s o}_{5}(\mathbf{C})$ as we see, for instance, by looking at their root systems. So the only possible factors in $S$ are, up to isogeny, $\mathrm{SL}_{2}(\mathbf{C}), \mathrm{SL}_{2}(\mathbf{R})$, and $\mathrm{SO}_{3}(\mathbf{R})$. By [21, $\S 5$, Theorem 4], the image of $\Gamma$ in $N / R_{u}$ is Haagerup, so that the image of $X$ in $H / R_{u}$ is finite.

(e) We keep the assumptions of (d), and suppose moreover that $X=\Lambda$ is a subgroup. Since $\Lambda$ is relatively compact, and $R_{u}$ is unipotent, $\Lambda \cap R_{u}=\{1\}$. Since we proved in (d) that the image of $\Lambda$ in $N / R_{u}$ is finite, this implies that $\Lambda$ is finite.

Now let us drop the assumption $Z=1$. Then the image of $\Lambda$ modulo $Z$ is finite, so that, by the case $Z=1, \Lambda$ is virtually contained in $Z$. This implies that $\Lambda$ is discrete, hence finite since it is also relatively compact.

In view of (c), (d), and (e), (2) is now proved; observe that (1) is an immediate consequence of (2) and (3).

(f) Now suppose that $\mathfrak{g} \simeq \mathfrak{s u}(2,1)$, and let us prove (4). Observe that (5) is an immediate consequence of (3) and (4).

We first suppose that $Z=1$, and that $\Gamma$ is not Zariski dense. So we continue with the notation of (d). Write $S=S_{c} S_{n c}$ by separating compact and noncompact simple factors.

Suppose that $S_{c} \neq 1$. This is a compact subgroup, up to conjugate, we can suppose that it is contained in the maximal subgroup $\operatorname{PS}(\mathrm{U}(2) \times \mathrm{U}(1))$. The Lie algebra of $S_{c}$ is identified with $\mathfrak{s u}(2)$.

CLAIM 4.6.2. - The only proper subalgebra of $\mathfrak{s u}(2,1)$ properly containing $\mathfrak{s u}(2)$ is $\mathfrak{s}(\mathfrak{u}(2) \times \mathfrak{u}(1))$.

Let us prove the claim. Let $\mathfrak{k}$ be such a subalgebra. If $\mathfrak{k} \subset \mathfrak{s}(\mathfrak{u}(2) \times \mathfrak{u}(1))$, then $\mathfrak{k}=$ $\mathfrak{s}(\mathfrak{u}(2) \times \mathfrak{u}(1))$ by a dimension argument.

Otherwise, we claim that the action of $\mathfrak{k}$ on $\mathbf{C}^{3}$ is irreducible. Let us consider the decomposition $\mathbf{C}^{3}=\mathbf{C}^{2} \oplus \mathbf{C}$. Let $A$ be the $\mathbf{C}$-subalgebra of $\mathbf{M}_{3}(\mathbf{C})$ generated by $\mathfrak{k}$. Since the action of $\mathfrak{s u}(2)$ on $\mathbf{C}^{2}$ is irreducible, $A$ contains $\mathrm{M}_{2}(\mathbf{C}) \times \mathrm{M}_{1}(\mathbf{C})$. In particular, the only possible stable subspaces are $\mathbf{C}^{2} \oplus\{0\}$ and $\{0\} \oplus\{0\} \oplus \mathbf{C}$. Now observe that since they are orthogonal to each other, if one is stable by $\mathfrak{k}$, then so is the other. So, if $\mathfrak{k}$ does not act irreducibly, it preserves these subspace; this means that $\mathfrak{k} \subset \mathfrak{s}(\mathfrak{u}(2) \times \mathfrak{u}(1))$. This proves the claim.

By the claim, $N$ is virtually isomorphic to a connected Lie group locally isomorphic to either $\mathrm{SO}_{3}(\mathbf{R})$ or $\mathrm{SO}_{3}(\mathbf{R}) \times \mathbf{R}$. So, by [21, 55 , Theorem 4], $\Gamma$ is Haagerup.

Otherwise, $S_{c}=1$. Since $G$ is Haagerup, by [10, Chapter 4] (or Corollary 3.3.4), $\left[S_{n c}, R_{u}\right]=$ $\{1\}$ so that $S_{n c}$ is, up to a finite kernel, a direct factor of $N$. Since we have proved in (d) that the only possible simple factor appearing in $S_{n c}$ are locally isomorphic to $\mathrm{SL}_{2}(\mathbf{R})$ or $\mathrm{SL}_{2}(\mathbf{C}){ }^{7}$ in view of $[21, \S 5$, Theorem 4], this implies that $\Gamma$ has a subgroup of finite index having the Haagerup Property, so that $\Gamma$ is Haagerup.

Finally let us drop the hypothesis $Z=1$. Let $N$ be the preimage in $G$ of the Zariski closure of $\Gamma$ in $G / Z$. There are two possible cases:

${ }^{7}$ Actually, it is easily checked that $\mathfrak{s l}_{2}(\mathbf{C})$ does not embed in $\mathfrak{s u}(2,1)$. 
- $N$ has finitely many connected components. Then, by [12, Theorem 3.13] (which relies on similar arguments), every subgroup of $N$ is Haagerup for the discrete topology.

- $N$ has infinitely many connected components. Then $N$ is almost the direct product of $Z$ and $N / Z$, so that, by the case $Z=1$, every subgroup of $N$ is Haagerup for the discrete topology.

The following proposition shows that the statements in Theorem 4.6.1 are, in a certain sense, optimal: in (1), the assumption that $\Lambda$ be a normal subgroup cannot be dropped, etc.

PROPOSITION 4.6.3. - Let $G$ be a connected Lie group, locally isomorphic to either $\mathrm{SO}(4,1)$ or $\mathrm{SU}(2,1)$.

(1) G has finitely presented subgroups $\Gamma \supset \Lambda$, such that $\Lambda$ is infinite and $(\Gamma, \Lambda)$ has relative Property (T).

(2) If $G$ is locally isomorphic to $\mathrm{SO}(4,1)$, then $G$ has a finitely presented subgroup $\Gamma$ and an infinite normal subset $X \subset \Gamma$ such that $(\Gamma, X)$ has relative Property (T).

Proof. - (1) First suppose that $G=\mathrm{SU}(2,1)$, and write $G=H(\mathbf{R})$, where $H(R)$ is defined, for every commutative ring $R$ as the set of matrices $(A, B)$ (rather denoted $A+i B$ ) satisfying the relation $\left({ }^{t} A-i^{t} B\right) J(A+i B)=J$, where ${ }^{8} J$ is the diagonal matrix $\operatorname{diag}(1,1,-1)$. Let $K$ be defined as the upper-left $2 \times 2$ block in $H$, so that $K(\mathbf{R}) \simeq \mathrm{SU}(2)$. Observe that $H(\mathbf{C}) \simeq \mathrm{SL}_{3}(\mathbf{C})$ and $K(\mathbf{C}) \simeq \mathrm{SL}_{2}(\mathbf{C})$.

Then $\Gamma=H\left(\mathbf{Z}\left[2^{1 / 3}\right]\right)$ embeds as a lattice in $H(\mathbf{R}) \times H(\mathbf{C})$. By Theorem 4.3.1, the projection $p$ of $\Gamma$ into $H(\mathbf{R}) \simeq \mathrm{SU}(2,1)$ is a resolution. Set $\Lambda=K\left(\mathbf{Z}\left[2^{1 / 3}\right]\right)$. Then $\Lambda$ is a lattice in $K(\mathbf{R}) \times K(\mathbf{C})$, so embeds as a cocompact lattice in $K(\mathbf{C}) \simeq \mathrm{SL}_{2}(\mathbf{C})$. On the other hand, since $p(\Lambda)$ is relatively compact (it is dense in $\mathrm{SU}(2)$ ), by Proposition 4.2.6, $(\Gamma, \Lambda)$ has relative Property $(\mathrm{T})$. Note that, as lattices in connected Lie groups, they are finitely presentable.

Let us now suppose that $G$ is locally isomorphic to $\mathrm{SU}(2,1)$, and let $Z$ be its centre. Let $\Gamma, \Lambda$ be as above, and let $\Gamma_{0}, \Lambda_{0}$ be their projection in $G / Z \times \mathrm{SL}_{3}(\mathbf{C})$. Finally, let $\Gamma_{1}, \Lambda_{1}$ be their preimage in $G \times \mathrm{SL}_{3}(\mathbf{C})$. If $Z$ is finite, then it is immediate that $\left(\Gamma_{1}, \Lambda_{1}\right)$ has relative Property (T), and that they are finitely presented. So we suppose that $G=\widetilde{\mathrm{SU}(2,1)}$. Let $q$ be the projection $G \times \mathrm{SL}_{3}(\mathbf{C}) \rightarrow \mathrm{SU}(2,1) \times \mathrm{SL}_{2}(\mathbf{C})$, and observe that $\Gamma_{1}=q^{-1}(\Gamma)$ and $\Lambda_{1}=q^{-1}(\Lambda)$.

Since $K(\mathbf{R}) \times K(\mathbf{C}) \simeq \mathrm{SU}(2) \times \mathrm{SL}_{2}(\mathbf{C})$ is simply connected, $W=q^{-1}(K(\mathbf{R}) \times K(\mathbf{C}))$ is isomorphic to $K(\mathbf{R}) \times K(\mathbf{C}) \times \mathbf{Z}$, and contains $\Lambda_{1}$ as a lattice. So we can define $\Lambda_{2}$ as the projection of $\Lambda_{1}$ into the unit component $W_{0}$, which is isomorphic to $\Lambda$, hence finitely presentable. Since the projection of $\Lambda_{2}$ on $G=K(\mathbf{R})$ is relatively compact, by Proposition 4.2.6, $\left(\Gamma_{1}, \Lambda_{2}\right)$ has relative Property ( $\left.\mathrm{T}\right)$.

A similar example can be constructed in $\mathrm{SO}(4,1)$, projecting an irreducible lattice from $\mathrm{SO}(4,1) \times \mathrm{SO}_{5}(\mathbf{C})$. Since $\mathrm{SO}(4,1)$ has finite fundamental group, we do not have to care with some of the complications of the previous example.

(2) Observe that $\mathrm{SO}(4,1)$ has a subgroup isomorphic to $\mathrm{SO}_{3}(\mathbf{R}) \ltimes \mathbf{R}^{3}$. Indeed, if we write $\mathrm{SO}(4,1)$ as $\left\{A \mid{ }^{t} A J A=J, \operatorname{det}(A)=1\right\}$, where $J=\left(\begin{array}{ccc}0 & 0 & 1 \\ 0 & I_{3} & 0 \\ 1 & 0 & 0\end{array}\right)$, then it contains the following subgroup, which is isomorphic to $\mathrm{SO}_{3}(\mathbf{R}) \ltimes \mathbf{R}^{3}$ :

$$
P=\left\{\left(\begin{array}{ccc}
1 & -{ }^{t} v A & -{ }^{t} v v / 2 \\
0 & A & v \\
0 & 0 & 1
\end{array}\right) \mid A \in \operatorname{SO}(3), v \in \mathbf{R}^{3}\right\} .
$$

\footnotetext{
${ }^{8}$ This relation must be understood as a relation where $i$ is a formal variable satisfying $i^{2}=-1$. In other words, this means ${ }^{t} A J A+{ }^{t} B J B=J$ and ${ }^{t} A J B-{ }^{t} B J A=0$.

$4^{\mathrm{e}}$ SÉRIE - TOME $39-2006-\mathrm{N}^{\circ} 2$
} 
Now consider the subgroup $\Gamma=\mathrm{SO}_{3}\left(\mathbf{Z}\left[2^{1 / 3}\right]\right) \ltimes \mathbf{Z}\left[2^{1 / 3}\right]^{3}$. Then $\Gamma$ embeds as a lattice in $\left(\mathrm{SO}_{3}(\mathbf{C}) \ltimes \mathbf{C}^{3}\right) \times\left(\mathrm{SO}_{3}(\mathbf{R}) \ltimes \mathbf{R}^{3}\right)$. By Theorem 3.3.1, $\left(\left(\mathrm{SO}_{3}(\mathbf{C}) \ltimes \mathbf{C}^{3}\right) \times\left(\mathrm{SO}_{3}(\mathbf{R}) \ltimes\right.\right.$ $\left.\mathbf{R}^{3}\right), \mathbf{C}^{3}$ ) has relative Property (T). Therefore, by Theorem 4.3.1, the inclusion morphism $\Gamma \rightarrow \mathrm{SO}_{3}\left(\mathbf{Z}\left[2^{1 / 3}\right]\right) \ltimes \mathbf{R}^{3}$ is a resolution. Let $B$ be the Euclidean unit ball in $\mathbf{R}^{3}$. Then, by Proposition 4.2.6, $\left(\Gamma, \mathbf{Z}\left[2^{1 / 3}\right]^{3} \cap B\right)$ has relative Property (T). Finally observe that $X=$ $\mathbf{Z}\left[2^{1 / 3}\right]^{3} \cap B$ is a normal subset in $\Gamma$.

Now observe that $\Gamma$ is contained in $P$, hence is contained in the unit component $\mathrm{SO}_{0}(4,1)$. The only other connected Lie group with Lie algebra $\mathfrak{s o}(4,1)$ is its universal covering (of degree 2); taking the preimage of $\Gamma$ and $X$, we obtain the required pair with relative Property (T).

Remark 4.6.4. - Examples similar to $\Gamma=\mathrm{SO}_{3}\left(\mathbf{Z}\left[2^{1 / 3}\right]\right) \ltimes \mathbf{Z}\left[2^{1 / 3}\right]^{3}$ (see the proof of Proposition 4.6.3) were already introduced in [12]. It was observed there that they provide the first known examples of groups without the Haagerup Property having no infinite subgroup with relative Property (T). We have made here more concrete the negation of the Haagerup Property by exhibiting an infinite subset with relative Property (T).

\subsection{Affine resolutions}

Although they are probably known to the specialists, we found no reference for the following lemmas.

LEMMA 4.7.1. - Let $M$ be a complete CAT(0) metric space. Let $X$ be a nonempty bounded subset, and let $B^{\prime}(c, r)$ be the closed ball of minimal radius containing $X$ [7, Chapter II, Corollary 2.8(1)]. Suppose that $X$ is contained in another ball $B^{\prime}\left(c^{\prime}, r^{\prime}\right)$. Then

$$
d\left(c, c^{\prime}\right)^{2} \leqslant r^{\prime 2}-r^{2} .
$$

Proof. - Set $d=d\left(c, c^{\prime}\right)$. Suppose the contrary, so that $d^{2}>r^{\prime 2}-r^{2} \geqslant 0$. For $t \in[0,1]$, set $p_{t}=(1-t) c+t c^{\prime}$, which is a well-defined point on the geodesic segment $\left[c c^{\prime}\right]$.

By [8, (**) p. 153], for every $z \in M$ and every $t \in[0,1]$,

$$
d\left(z, p_{t}\right)^{2} \leqslant(1-t) d(z, c)^{2}+t d\left(z, c^{\prime}\right)^{2}-t(1-t) d^{2} .
$$

It follows that if $z \in B^{\prime}(c, r) \cap B^{\prime}\left(c^{\prime}, r^{\prime}\right)$, then $d\left(z, p_{t}\right)^{2} \leqslant(1-t) r^{2}+t r^{\prime 2}-t(1-t) d^{2}$; denote by $u(t)$ this expression. By an immediate calculation, $u(t)$ is minimal for $t=t_{0}=$ $\left(d^{2}+r^{2}-r^{\prime 2}\right) /\left(2 d^{2}\right)$, which belongs to $\left.] 0,1\right]$ by assumption. Since $u(0)=r^{2}$, it follows that $u\left(t_{0}\right)<r^{2}$. Since this is true for all $z \in B^{\prime}(c, r) \cap B^{\prime}\left(c^{\prime}, r^{\prime}\right)$, this implies that $X$ is contained in a closed ball of radius $u\left(t_{0}\right)^{1 / 2}<r$, contradiction.

LEMMA 4.7.2. - Let $M$ be a complete CAT(0) metric space. Let $K$ be a nonempty closed convex bounded subset, and $B^{\prime}(c, r)$ the ball of minimal radius containing $K$. Then $c \in K$.

Proof. - Suppose that $c \notin K$, and let $p$ be its projection on $K$ [7, Chapter II, Proposition 2.4]. Fix $x \in K$. Then for every $p^{\prime} \in[p x], d(p, c) \leqslant d\left(p^{\prime}, c\right)$. Hence, by (4.1), for all $t \in[0,1]$, $d(p, c)^{2} \leqslant(1-t) d(p, c)^{2}+t d(x, c)^{2}-t(1-t) d(p, x)^{2}$. Taking the limit, after dividing by $t$, when $t \rightarrow 0$, gives $d(x, p)^{2} \leqslant d(x, c)^{2}-d(p, c)^{2}$, so that $d(x, p)^{2} \leqslant r^{2}-d(p, c)^{2}$. In other words, $K \subset B^{\prime}\left(p,\left(r^{2}-d(p, c)^{2}\right)^{1 / 2}\right)$. This contradicts the minimality of $r$.

LEMmA 4.7.3. - Let $M$ be a complete CAT $(0)$ metric space. Let $\left(F_{n}\right)$ be a decreasing sequence of nonempty closed convex bounded subsets. Then $\bigcap F_{n} \neq \emptyset$.

Proof. - Let $B^{\prime}\left(c_{n}, r_{n}\right)$ be the ball of minimal radius containing $F_{n}$. Observe that $c_{n} \in F_{n}$ by Lemma 4.7.2. Moreover, $\left(r_{n}\right)$ is non-increasing, hence converges. 
On the other hand, if $m \leqslant n$, then $F_{n} \subset F_{m}$. Applying Lemma 4.7.1, we get $d\left(c_{n}, c_{m}\right)^{2} \leqslant$ $r_{n}^{2}-r_{m}^{2}$. Therefore, $\left(c_{n}\right)$ is Cauchy, hence has a limit $c$, which belongs to $\bigcap F_{n}$.

THEOREM 4.7.4. - Let $f: G \rightarrow Q$ be a morphism with dense image between locally compact groups. Let $G$ act by isometries on a complete $\mathrm{CAT}(0)$ metric space $M$. Suppose that there exists a neighbourhood $\Omega$ of 1 in $Q$, such that, for some $w \in M, f^{-1}(\Omega) w$ is bounded. Then $M^{Q}$ is nonempty.

Proof. - Let $\left(\Omega_{n}\right)$ be a sequence of compact symmetric neighbourhoods of 1 in $Q$, contained in $\Omega$, such that $\Omega_{n+1} \cdot \Omega_{n+1} \subset \Omega_{n}$ for all $n$. Set $V_{n}=f^{-1}\left(\Omega_{n}\right)$.

By the assumption on $\Omega, V_{n} \cdot w$ is bounded for all $n$. Let $B^{\prime}\left(c_{n}, r_{n}\right)$ be the minimal ball containing $V_{n} \cdot w$. Note that the sequence $\left(c_{n}\right)$ is bounded since $d\left(c_{n}, w\right) \leqslant r_{n} \leqslant r_{0}$ for all $n$.

Then, for all $g \in V_{n+1}$, we have $g^{-1} V_{n+1} \cdot w \subset V_{n} \cdot w \subset B^{\prime}\left(c_{n}, r_{n}\right)$, so that $V_{n+1} \cdot w \subset$ $B^{\prime}\left(g c_{n}, r_{n}\right)$. By Lemma 4.7.1, we have

$$
d\left(c_{n+1}, g c_{n}\right) \leqslant \sqrt{\left(r_{n}-r_{n+1}\right)\left(r_{n}+r_{n+1}\right)} \leqslant \sqrt{2 r_{0}\left(r_{n}-r_{n+1}\right)} .
$$

Specializing this inequality to $g=1$, we obtain

$$
d\left(c_{n+1}, c_{n}\right) \leqslant \sqrt{2 r_{0}\left(r_{n}-r_{n+1}\right)},
$$

and combining the two previous inequalities, we get, for all $g \in V_{n+1}$,

$$
d\left(c_{n}, g c_{n}\right) \leqslant d\left(c_{n+1}, c_{n}\right)+d\left(c_{n+1}, g c_{n}\right) \leqslant 2 \sqrt{2 r_{0}\left(r_{n}-r_{n+1}\right)} .
$$

Set $u(n)=\sup \left\{2 \sqrt{2 r_{0}\left(r_{m}-r_{m+1}\right)} \mid m \geqslant n\right\}$. Since $\left(r_{n}\right)$ is non-increasing and nonnegative, $r_{n}-r_{n+1} \rightarrow 0$, so that $u(n) \rightarrow 0$.

Note that, for all $g$, the function $x \mapsto d(x, g x)$ is continuous and convex on $M$ [7, Chapter II, Proposition 6.2]. It follows that $F_{n}=\left\{v \in \mathscr{H} \mid \forall g \in V_{n+1}, d(v, g v) \leqslant u(n)\right\}$ is closed and convex. Set $K_{n}=F_{n} \cap B^{\prime}\left(w, r_{0}\right)$. Then $\left(K_{n}\right)$ is a decreasing sequence of closed, convex, bounded subsets of $M$, nonempty since $c_{n} \in K_{n}$. By Lemma 4.7.3, $\bigcap K_{n}$ is nonempty; pick a point $y$ in the intersection. We claim that $y \in X^{Q}$ : to see this, let us appeal to Proposition 4.1.3. Let $g_{i}$ be a net in $G$ such that $f\left(g_{i}\right) \rightarrow 1$. Set $n_{i}=\sup \left\{n \mid g_{i} \in \Omega_{n+1}\right\} \in \mathbf{N} \cup\{\infty\}$. Then $n_{i} \rightarrow \infty$ since all $\Omega_{n}$ are neighbourhoods of 1 in $Q$, and $d\left(y, g_{i} y\right) \leqslant u\left(n_{i}\right)$ for all $i$ (where we set $u(\infty)=0)$. It follows that $d\left(y, g_{i} y\right) \rightarrow 0$. By Proposition 4.1.3, $y \in X^{Q}$.

Definition 4.7.5. - Let $f: G \rightarrow Q$ be a morphism with dense image between locally compact groups. We call it an affine resolution if, for every isometric action of $G$ on an affine Hilbert space, there exists a nonempty $G$-invariant affine subspace such that the action of $G$ on this subspace factors through $Q$.

THEOREM 4.7.6. - Let $G, Q$ be locally compact groups, $f: G \rightarrow Q$ be a morphism with dense image. Consider the following conditions:

(1) $G \rightarrow Q$ is a resolution.

(2) $(G, X)$ has relative Property (T) for all subsets $X \subset G$ such that $\overline{f(X)}$ is compact.

(3) $(G, X)$ has relative Property $(\mathrm{FH})$ for all subsets $X \subset G$ such that $f(X)$ is compact.

(4) $G \rightarrow Q$ is an affine resolution.

Then the implications (1) $\Rightarrow(2) \Rightarrow(3) \Leftrightarrow$ (4) hold. Moreover, if $G$ is $\sigma$-compact, then (4) $\Rightarrow(1)$, so that they are all equivalent.

Proof. - (3) is an immediate consequence of (4). The converse actually follows immediately from Theorem 4.7.4. The implication $(1) \Rightarrow(2)$ has been proved in Proposition 4.2.6, and 
(2) $\Rightarrow$ (3) follows from Theorem 2.2.3. It remains to prove (4) $\Rightarrow$ (1). Hence, suppose that $G$ (hence $Q$ ) is $\sigma$-compact, and that $G \rightarrow Q$ is an affine resolution.

CLAIM 4.7.7. - For every unitary representation $\pi$ of $G$ such that $1_{G} \prec \pi, \pi^{Q} \neq 0$.

Let us prove the claim. Let $\pi$ be a unitary representation of $G$ on a Hilbert space $\mathscr{H}$, such that $1_{G} \prec \pi$. We must show that $\pi^{Q} \neq 0$. If $1 \leqslant \pi$, this is trivially satisfied. So we can suppose that $1 \nless \pi$. By a result of Guichardet which uses $\sigma$-compactness (see [3, Theorem 2.13.2]), $B^{1}(G, \pi)$ is not closed in $Z^{1}(G, \pi)$, so that, in particular, $H^{1}(G, \pi) \neq 0$. Consider $b \in Z^{1}(G, \pi)-B^{1}(G, \pi)$, and let $\alpha$ be the associated affine action. Since $f$ is an affine resolution, $\alpha^{Q}$ is a nonempty closed affine subspace $V$ of $\mathscr{H}$. Then $V$ is not reduced to a point $\{v\}$ : otherwise, $v$ would be a fixed point for the action of $G$, contradicting $b \notin B^{1}(G, \pi)$. Hence the linear part of $\alpha^{Q}$ is a nonzero subrepresentation of $\pi$, so that $\pi^{Q}$ is nonzero. This proves the claim.

Let $\pi$ be a unitary representation of $G$ on a Hilbert space $\mathscr{H}$, such that $1_{G} \prec \pi$. We must show that $1_{Q} \prec \pi^{Q}$. Again, since the case when $1 \leqslant \pi$ is trivial, we suppose that $1 \nless \pi$. Let $\rho$ be the orthogonal of $\pi^{Q}$. By the claim, $1_{G} \nprec \rho$. It follows that $1_{G} \prec \pi^{Q}$, so that we can suppose that $\pi=\pi^{Q}$, i.e. $\pi$ factors through a representation $\tilde{\pi}$ of $Q$.

CLAIM 4.7.8. - The natural continuous morphism $\hat{f}: Z^{1}(Q, \tilde{\pi}) \rightarrow Z^{1}(G, \pi)$ is bijective.

Let us prove the claim. The morphism $\hat{f}$ is clearly injective. Take $b \in Z^{1}(G, \pi)$. Since $f$ is an affine resolution, one can write $b(g)=b^{\prime}(g)+\pi(g) v-v(\forall g \in G)$, where $b^{\prime} \in Z^{1}(G, \pi)$ factors through $Q$ and $v \in \mathscr{H}$. Since $\pi$ also factors through $Q$, this implies that $b$ does so, which means that $b$ belongs to $\operatorname{Im}(\hat{f})$, and the claim is proved.

Since $G$ and $Q$ are $\sigma$-compact, $Z^{1}(G, \pi)$ and $Z^{1}(Q, \tilde{\pi})$ are Fréchet spaces. Since $\hat{f}: Z^{1}(Q, \tilde{\pi}) \rightarrow Z^{1}(G, \pi)$ is bijective, by the open mapping theorem, it is an isomorphism. Note that it maps $B^{1}(Q, \tilde{\pi})$ bijectively onto $B^{1}(G, \pi)$, and that $B^{1}(G, \pi)$ is not closed in $Z^{1}(G, \pi)$, as we used in the proof of the first claim. It follows that $B^{1}(Q, \tilde{\pi})$ is not closed in $Z^{1}(Q, \tilde{\pi})$. Using the converse in Guichardet's result [3, Theorem 2.13.2], $1_{Q} \prec \tilde{\pi}$.

As a corollary of Theorems 4.7.4 and 4.7.6, we get:

COROLLARY 4.7.9. - Let $G, Q$ be locally compact groups, and let $f: G \rightarrow Q$ be a resolution. Let $G$ act isometrically on a complete $\mathrm{CAT}(0)$ metric space $M$, and suppose that there exists a $G$-equivariant proper embedding $i$ of $M$ into a Hilbert space. Then $M^{Q} \neq \emptyset$.

Proof. - Let $\Omega$ be a compact neighbourhood of 1 in $Q$, and set $V=f^{-1}(\Omega)$. Fix $x \in M$, and set $\psi(g)=\|i(g x)\|^{2}$. Then $\psi$ is conditionally negative definite on $G$. By Proposition 4.2.6, $\psi$ is bounded on $V$. This implies that the hypothesis of Theorem 4.7.4 is fulfilled.

There are many metric spaces for which there automatically exists such an equivariant embedding; namely, those metric spaces $M$ that have a $\operatorname{Isom}(M)$-equivariant embedding in a Hilbert space. Thus the hypotheses of corollary are satisfied, for instance when

- $M$ is a Hilbert space,

- $M$ is a tree, or a complete $\mathbf{R}$-tree [22, Chapter 6, Proposition 11].

- $M$ is a real or complex hyperbolic space (maybe infinite-dimensional) [18],

- $M$ is a finite-dimensional CAT(0) cube complex [32].

For instance, we have

COROLlary 4.7.10. - Let $G, Q$ be locally compact groups, and let $f: G \rightarrow Q$ be a resolution. Then $G$ has Property (FA) if and only if $Q$ does so.

Proof. - If $G$ has Property (FA), so does $Q$. Let us show the converse. By a result of Alperin and Watatani (see [22, Chapter 6]), every tree equivariantly embeds in a Hilbert space (more 
precisely, the distance is a conditionally negative definite kernel). It follows that, for every isometric action of $G$ on a tree, there exists a nonempty $G$-invariant subtree on which the action factors through $Q$. The result immediately follows.

Theorem 4.7.6 allows us to prove the converse of Corollary 4.2.5.

THEOREM 4.7.11. - Let $f: G \rightarrow Q$ be a morphism between locally compact groups, with dense image, and suppose $G \sigma$-compact. Then $f$ is a resolution if and only if, for every net $\left(\pi_{i}\right)$ of irreducible unitary representations of $G$ converging to $1_{G}$, eventually $\pi_{i}$ factors through a representation $\tilde{\pi}_{i}$ of $Q$, and $\tilde{\pi}_{i} \rightarrow 1_{Q}$.

Proof. - The condition is necessary by Corollary 4.2.5. Conversely suppose that it is satisfied. Let us show that (2) of Theorem 4.7.6 is satisfied, using Theorem 2.3.3. Fix $\varepsilon>0$, let $X \subset G$ be a subset such that $\overline{f(X)}$ is compact, and let $\left(\pi_{i}\right)$ be a net of irreducible unitary representations of $G$ converging to $1_{G}$. Then eventually $\pi_{i}$ factors through a representation $\tilde{\pi}_{i}$ of $Q$, and $\tilde{\pi}_{i} \rightarrow 1_{Q}$. Since $\overline{f(X)}$ is compact, this implies that, eventually, $\tilde{\pi}_{i}$ has a $(\overline{f(X)}, \varepsilon)$-invariant vector, so that $\pi_{i}$ has a $(X, \varepsilon)$-invariant vector.

\section{Acknowledgements}

I thank Bachir Bekka, Pierre de la Harpe, Vincent Lafforgue, Romain Tessera, and Alain Valette for valuable comments and useful discussions.

\section{REFERENCES}

[1] Akemann C.A., Walter M.E., The Riemann-Lebesgue property for arbitrary locally compact groups, Duke Math. J. 43 (1976) 225-236.

[2] Akemann C.A., Walter M.E., Unbounded negative definite functions, Canad. J. Math. 33 (4) (1981) 862-871.

[3] BeKKa B., De la Harpe P., Valette A., Kazhdan's Property (T), Forthcoming book, currently available at http://name.math.univ-rennes1.fr/bachir.bekka/, 2004.

[4] BEKKA B., Louvet N., On a variant of Kazhdan's Property (T) for subgroups of semisimple groups, Ann. Inst. Fourier 47 (4) (1997) 1065-1078.

[5] Borel A., Serre J.-P., Théorèmes de finitude en cohomologie galoisienne, Comment. Math. Helv. 39 (1964) 111-164.

[6] Borel A., Tits J., Groupes réductifs, Publ. Math. Inst. Hautes Études Sci. 27 (1965) 55-151.

[7] Bridson M.R., Haefliger A., Metric Spaces of Non-Positive Curvature, Springer, Berlin, 1999.

[8] BROWn K.S., Buildings, Springer, Berlin, 1989.

[9] Burger M., Kazhdan constants for $\mathrm{SL}_{3}(\mathbf{Z})$, J. Reine Angew. Math. 431 (1991) 36-67.

[10] Cherix P.-A., Cowling M., Jolissaint P., Julg P., Valette A., Groups with the Haagerup Property, Progress in Mathematics, vol. 197, Birkhäuser, Basel, 2001.

[11] Comfort W.W., Topological groups, in: Kunen K., Vaughan J.E. (Eds.), Handbook of Set-Theoretic Topology, North-Holland, Amsterdam, 1984, pp. 1143-1263.

[12] DE Cornulier Y., Kazhdan and Haagerup Properties in algebraic groups over local fields, J. Lie Theory 16 (2006) 67-82.

[13] DE CORNULIER Y., Strongly bounded groups and infinite powers of finite groups, Comm. Algebra, in press.

[14] DE CORnUlier Y., On Haagerup and Kazhdan Properties, Thèse sciences, École Polytechnique Fédérale de Lausanne, $\mathrm{N}^{\mathrm{o}} 3438$ (2005).

[15] De CORnUlier Y., Tessera R., VALETTE A., Isometric group actions on Hilbert spaces: growth of cocycles, Preprint $\mathrm{N}^{\mathrm{O}} 3438,2005$.

[16] Delaroche C., Kirillov A., Sur les relations entre l'espace dual d'un groupe et la structure de ses sous-groupes fermés (d'après Kajdan), Sém. Bourbaki, 20e année, 1967-1968, №343, 1968.

$4^{\text {e }}$ SÉRIE - TOME $39-2006-\mathrm{N}^{\circ} 2$ 
[17] DiXMIER J., Les C*-algèbres et leurs représentations, Gauthier-Villars, Paris, 1969.

[18] FARAUt J., HaRZallah K., Distances hilbertiennes invariantes sur un espace homogène, Ann. Inst. Fourier 24 (3) (1974) 171-217.

[19] FERnós T., Relative Property (T) and linear groups, arXiv math.GR/0411527, Ann. Inst. Fourier, in press.

[20] Furstenberg H., A note on Borel's density theorem, Proc. Amer. Math. Soc. 55 (1976) 209-212.

[21] Guentner E., Higson N., Weinberger S., The Novikov Conjecture for linear groups, Publ. Math. Inst. Hautes Études Sci. 101 (2005) 243-268.

[22] DE la Harpe P., VAletTe A., La propriété (T) de Kazhdan pour les groupes localement compacts, Astérisque, vol. 175, SMF, Paris, 1989.

[23] Joliss Aint P., On Property (T) for pairs of topological groups, Enseign. Math. (2) 51 (2005) 31-45.

[24] KaZHDAN D., Connection of the dual space of a group with the structure of its closed subgroups, Funct. Anal. Appl. 1 (1967) 63-65.

[25] LAFFORGUE V., Une remarque sur les fonctions conditionnellement de type négatif, C. R. Acad. Sci., in press.

[26] LUBOTZKY A., ZIMmeR R., Variants of Kazhdan's property for subgroups of semisimple groups, Israel J. Math. 66 (1989) 289-298.

[27] LubotZKY A., ŻUK A., On Property $(\tau)$, Forthcoming book, 2005.

[28] Margulis G., Finitely-additive invariant measures on Euclidean spaces, Ergodic Theory Dynam. Systems 2 (3-4) (1982) 383-396.

[29] Margulis G., Discrete Subgroups of Semisimple Lie Groups, Springer, Berlin, 1991.

[30] Martin F., Reduced 1-cohomology of connected locally compact groups and applications, J. Lie Theory 16 (2006) 311-328.

[31] Montgomery D., ZIPPIN L., Topological Transformation Groups, Interscience, New York, 1955.

[32] Niblo G., ReEves L., Groups acting on CAT(0) cube complexes, Geom. Topology 1 (1997) 1-7.

[33] Peterson J., Popa S., On the notion of relative property (T) for inclusions of von Neumann algebras, J. Funct. Anal. 219 (2005) 469-483.

[34] PopA S., Strong rigidity of $\mathrm{II}_{1}$ factors arising from malleable actions of w-rigid groups, II, math.OA/0407103, Invent. Math., in press.

[35] Shalom Y., Bounded generation and Kazhdan's property (T), Publ. Math. Inst. Hautes Études Sci. 90 (1999) 145-168.

[36] Shalom Y., Invariant measures for algebraic actions, Zariski dense subgroups and Kazhdan's property (T), Trans. Amer. Math. Soc. 351 (1999) 3387-3412.

[37] Shalom Y., Rigidity of commensurators and irreducible lattices, Invent. Math. 141 (1) (2000) 1-54.

[38] Tessera R., Asymptotic isoperimetry on groups and uniform embeddings into Banach spaces, Preprint, 2006.

[39] VAlette A., Group pairs with property (T), from arithmetic lattices, Geom. Dedicata 112 (1) (2005) 183-196.

[40] WANG S.P., On the Mautner phenomenon and groups with property (T), Amer. J. Math. 104 (6) (1982) 1191-1210.

(Manuscrit reçu le 16 mai 2005; accepté, après révision, le 16 décembre 2005.)

Yves DE CORNULIER

École Polytechnique Fédérale de Lausanne (EPFL),

Institut de Géométrie,

Algèbre et Topologie (IGAT),

CH-1015 Lausanne, Switzerland

E-mail: decornul@clipper.ens.fr 